\title{
IVAG: An Integrative Visualization Application for Various Types of Genomic Data Based on R-Shiny and the Docker Platform
}

\author{
Tae-Rim Lee ${ }^{\S}$, Jin Mo Ahn ${ }^{\S}$, Gyuhee Kim ${ }^{\S}$ Sangsoo Kim* \\ Department of Bioinformatics and Life Science, Soongsil University, Seoul 06978, Korea
}

\begin{abstract}
Next-generation sequencing (NGS) technology has become a trend in the genomics research area. There are many software programs and automated pipelines to analyze NGS data, which can ease the pain for traditional scientists who are not familiar with computer programming. However, downstream analyses, such as finding differentially expressed genes or visualizing linkage disequilibrium maps and genome-wide association study (GWAS) data, still remain a challenge. Here, we introduce a dockerized web application written in R using the Shiny platform to visualize pre-analyzed RNA sequencing and GWAS data. In addition, we have integrated a genome browser based on the JBrowse platform and an automated intermediate parsing process required for custom track construction, so that users can easily build and navigate their personal genome tracks with in-house datasets. This application will help scientists perform series of downstream analyses and obtain a more integrative understanding about various types of genomic data by interactively visualizing them with customizable options.
\end{abstract}

Keywords: docker, genome browser, genome-wide association study, RNA sequencing, Shiny, visualization

Availability: A docker image of IVAG can be downloaded at https://hub.docker.com/r/leetaerim/ivag/. Pre-processed example input data and the manual file are available at https://github.com/jmoa/IVAG.

\section{Introduction}

Since its advent, high throughput next-generation sequencing (NGS) technology has revolutionized the genomics research area, including transcriptome analysis and genomewide association studies (GWASs) taking advantage of accelerated sequencing speed with reduced cost $[1,2]$. Even though many bioinformatics software programs have been developed to handle and analyze the massive data generated from NGS, most of them are based on a command-line interface and require quite a high level of computational power [3], which creates a high barrier for wet lab biologists to enter into this field. Thanks to web-based analysis platforms, including Galaxy [4] and BIOEXPRESS [5], this barrier has been lowered. However, there still are problems. Downstream analyses, such as finding differentially expre- ssed genes (DEGs), conducting Gene Ontology (GO) enrichment analysis, calculating linkage disequilibrium (LD), annotating gene information into GWAS results, and finally visualizing the resulting data, still require significant computer programming skills.

In this study, we present a dockerized application, IVAG. It provides a user-friendly web interface in which all downstream analyses mentioned above can be carried out without any programming knowledge. Detailed parameters for each analysis step can be adjusted with simple click-and-drag operation. IVAG interactively outputs publication-quality plots in response to the given parameters, and all of these plots can be downloaded. Also, a variety of data types, ranging from RNA sequencing (RNA-seq) and GWAS results to sequence read alignments, gene annotation, variant call information, and peak information, can be uploaded into the embedded genome browser and then 
visualized together to help users gain greater integrative insights into their data. Furthermore, IVAG is lightweight, allowing it to be deployed on a desktop computer, as well as a server application.

\section{Methods}

IVAG is mostly written in the $\mathrm{R}$ programming language [6] and dockerized [7] with all required dependencies to avoid compatibility issues. It uses the Shiny package [8] to build a user-friendly web interface and several other packages to analyze and visualize RNA-seq and GWAS data (Supplementary Table 1). VCFtools [9] and PLINK (v1.90b4.6) [10] were used for the LD analysis. The JBrowse platform [11] was integrated into IVAG, and all intermediate steps required for custom track construction were automated using a custom BASH script. Three publicly available plugins (Supplementary Table 2), with slight modification, were incorporated into the genome browser to build GWAS, GC content, and browser extensible data (BED) tracks. Gene transfer format (GTF)-to-general feature format 3 (GFF3) format conversion was carried out with Cufflinks (2.2.1) [12]. Binary sequence alignment map (BAM) and variant call format (VCF) files were sorted with SAMtools [13]. Example data were prepared using publicly available RNA-seq and GWAS data (Supplementary Table 3).

\section{Results \\ Workflow}

Fig. 1 shows a graphical overview of the pre-analysis steps and the IVAG workflow. The blue line (Fig. 1A) denotes a schematic representation of the external pipelines required for RNA-seq and GWAS data. These parts are prerequisites for downstream analyses prior to IVAG analysis. The red box in the right panel (Fig. 1B) illustrates the IVAG workflow.
The orange items are input files for IVAG, and their detailed formats are described in Supplementary Figs. 1-29.

\section{RNA-seq}

IVAG DEG analysis requires raw count RNA sequence data that can be generated using open source software, such as Htseq [14]. DEG analysis outputs a DEG results table generated with user-specified parameters based on the $\mathrm{R}$ Bioconductor package edgeR [15]. The output table consists of multiple columns, such as log2 fold-change, $\log 2$ count per million, associated p-value, and associated false discovery rate (Fig. 2A), and it can be visualized as a heatmap, a volcano plot, and a principal component analysis plot (Fig. 2B). The heatmap is generated using raw count data, which are converted to counts per million and normalized to a have row-based percentage value. The volcano plot is generated using log2 fold-change and the associated false discovery rate. Principal component analysis is generated using the raw count of each sample normalized to the log2 count per million. The heatmap and volcano plot can be interactively updated based on user-specified filtering criteria, such as false discovery rate or absolute fold-change. GO enrichment analysis uses the DEG analysis results table, GO annotation file, and GTF file. The DEG analysis results table can be generated using IVAG DEG analysis, or a pre-analyzed DEG analysis result can also be used. The GO annotation file consists of two columns: gene ID and GO category. A GTF file is needed to generate the gene length of each gene in the DEG analysis table. However, it can be omitted if a user wishes not to take gene length bias into account. IVAG GO enrichment analysis outputs over-represented and underrepresented GO terms among DEGs (Fig. 2C) using the $\mathrm{R}$ Bioconductor package goseq [16]. It also shows a histogram of DEGs in each GO category based on its ontology: biological process, cellular component, and molecular function.

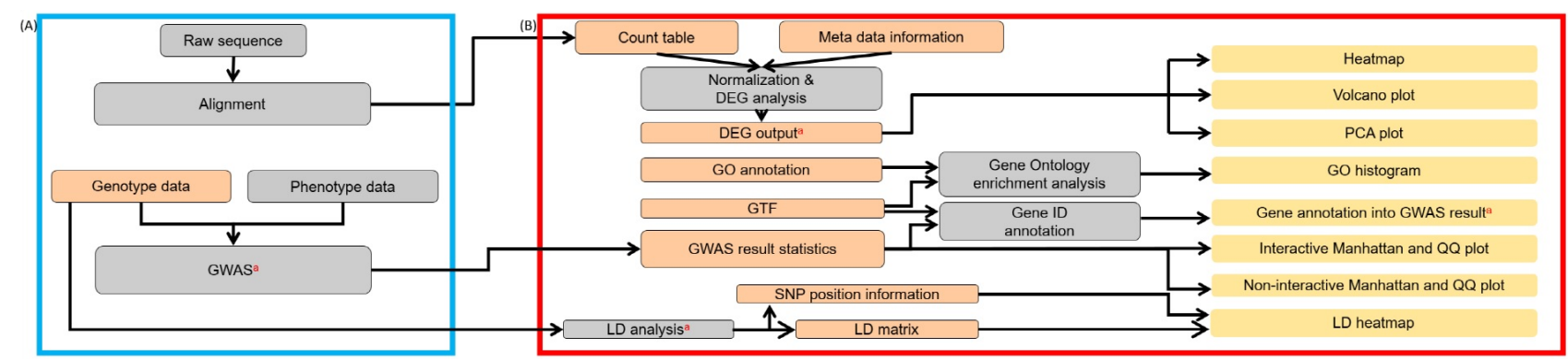

Fig. 1. Graphical overview of IVAG workflow. (A) External pre-calculation and automated pipelines for RNA sequencing and genome-wide association study (GWAS) analysis. (B) Schematic representation of the App pipeline. DEG, differentially expressed gene; GO, Gene Ontology; GTF, gene transfer format; SNP, single nucleotide polymorphism; LD, linkage disequilibrium; PCA, principal component analysis; QQ, quantile-quantile. ${ }^{\text {T}}$ These data can be uploaded directly to the genome browser. The orange items are input files for IVAG, while the yellow ones are output files. 

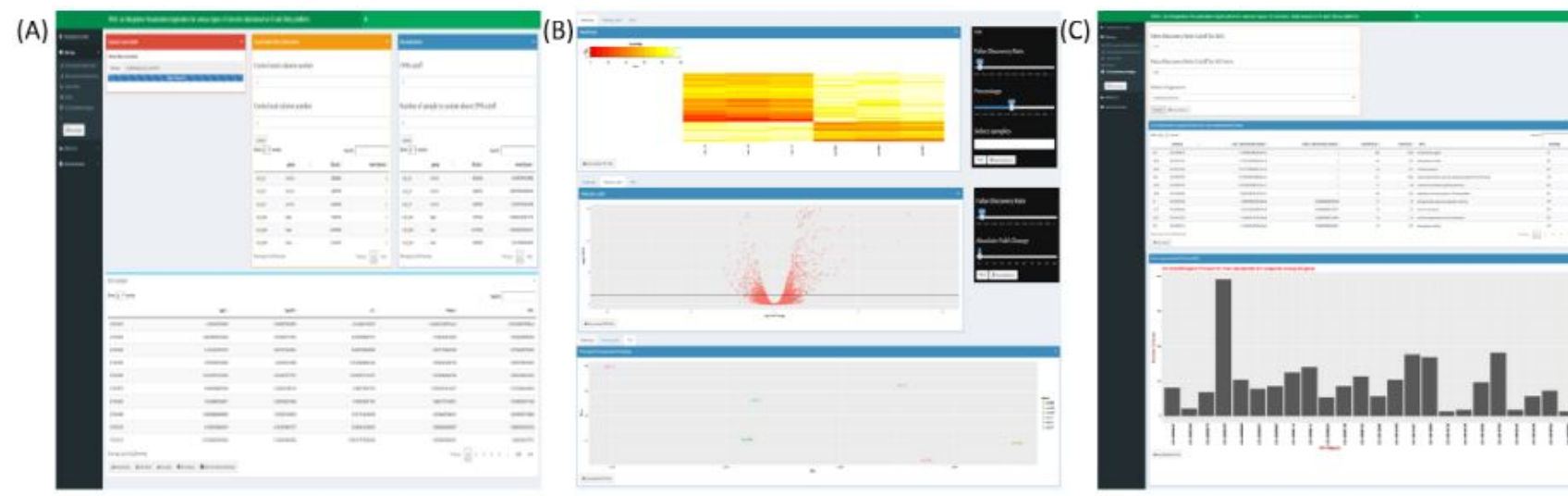

(D)

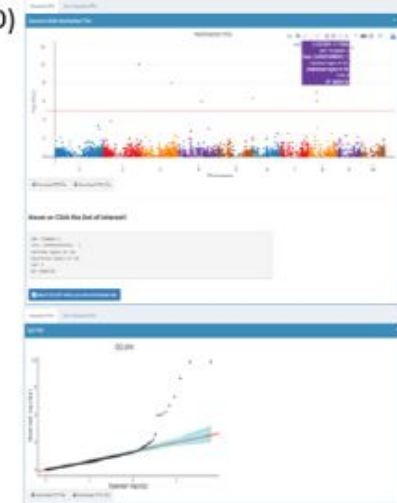

(E)

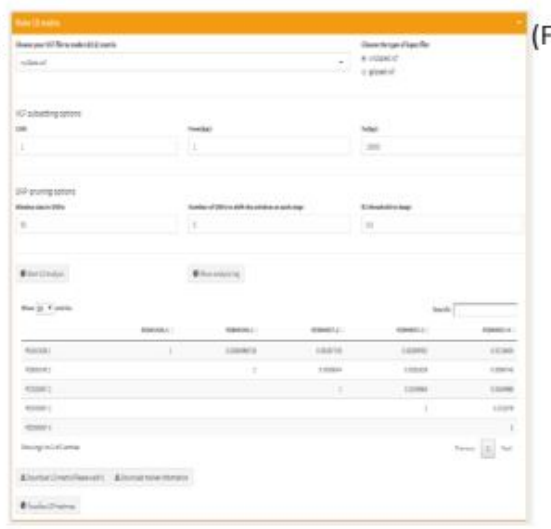

(F)

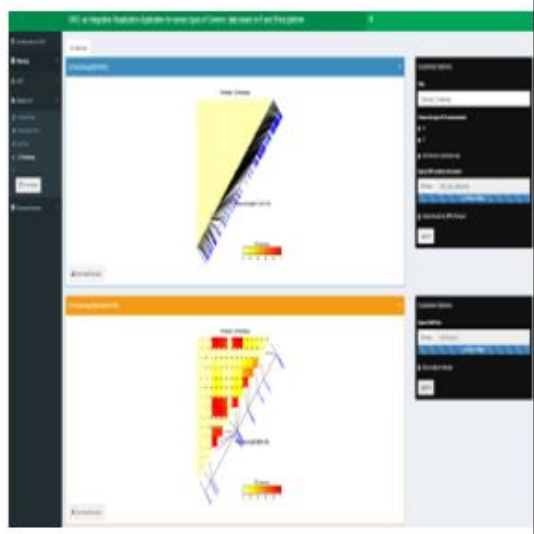

(G)

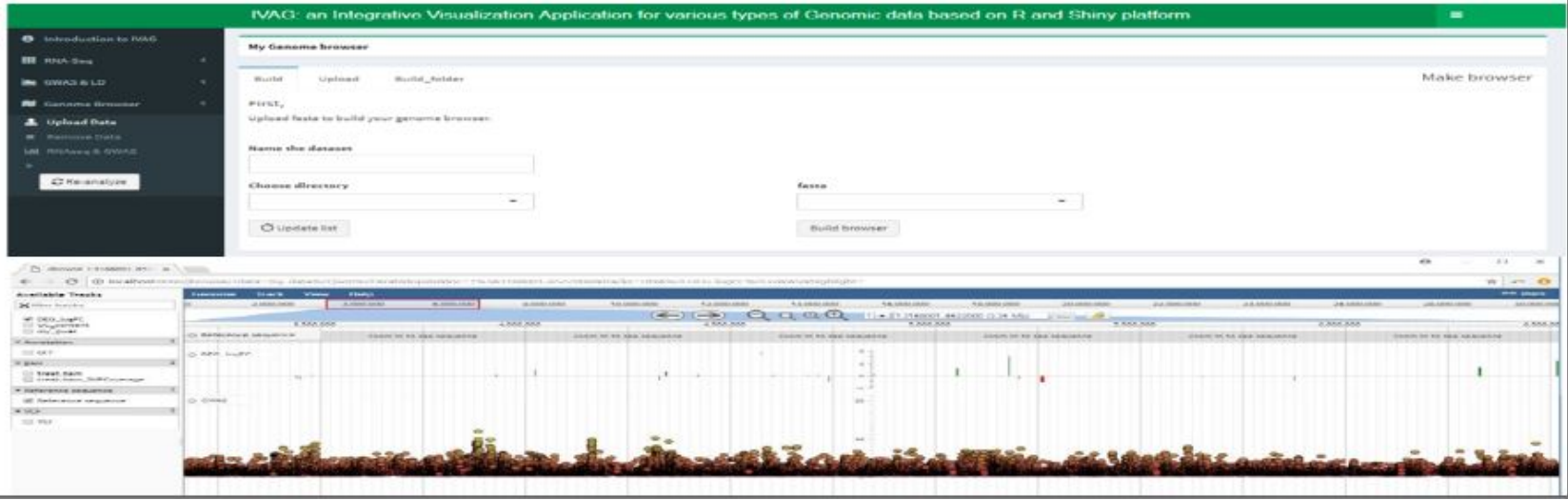

Fig. 2. Functions and results of IVAG. (A) Single-factor differential expression analysis. (B) Heatmap, volcano, and principal component analysis plot drawn with specified parameters. (C) Result of gene ontology enrichment analysis. Histogram shows how many differentially expressed genes are allocated to specific Gene Ontology categories. (D) Manhattan and quantile-quantile plots drawn with customizable options. (E) Linkage disequilibrium (LD) analysis generating LD matrix. (F) Pairwise LD heatmap. A group of single nucleotide polymorphisms of interest can be the subset. (G) Genome browser track with integrated view of differentially expressed gene and genome-wide association study results.

\section{GWAS}

Gene ID annotation requires a tab-separated GWAS result file comprising marker ID, chromosome ID, base position, and p-value columns in order, and a GTF file that contains strand and position information of genes. It returns a new GWAS result file in which gene, upstream, and downstream columns are added. Both GWAS result files, before and after this annotation, can be visualized in Manhattan and quantile-quantile plot with customizable options (Fig. 2D). One can see all information for a specific dot of one's interest if he clicks on the interactive plots. The LD analysis part is read in a VCF file with several detailed options to generate an LD matrix and a marker information file, which can be visualized in the LD heatmap (Fig. 2E and 2F). 


\section{Genome browser}

Constructing a custom genome browser with a reference genome sequence is the first step. After selecting one of the genome browsers configured in IVAG, various types of genomic data, including GTF, GFF3, BAM, BED, BigWig, and VCF, can be uploaded and visualized all together (Fig. 2G). Also, this genome browser receives RNA-seq and GWAS results generated from IVAG as inputs.

\section{Discussion}

IVAG is an easy-to-use, web-based application with three modules, including RNA-seq, GWAS, and a genome browser. This application enables scientists with little computational proficiency to analyze and visualize their data easily. Some web applications provide similar functions for RNA-seq and GWAS, but they have some limitations. For example, DEApp [17] provides differential expression analysis using three different methods-edgeR, limma-voom, and DESeq2while a heatmap or a principal component analysis plot is not provided. START [18] can output several plots, but it does not offer a GO enrichment analysis function. LocusTrack [19] can visualize GWAS data and annotate multiple tracks on them, but it is limited to only one species, human. Zbrowse [20] can be used over every species. However, because it focuses on plotting multiple GWAS results in one panel to enable users to detect genotype-environment interactions, the number of markers that can be plotted for one trait is limited to 5,000 . IVAG is not limited to a specific organism or the number of markers [14]. Most importantly, IVAG combines a genome browser with analysis and visualization modules so that users can analyze, visualize, and finally navigate their entire data interactively in one application. We offer only two analysis and visualization modules now, but several more modules are in development and will be included in the near future.

ORCID: Tae-Rim Lee: http://orcid.org/0000-0003-06846552; Jin Mo Ahn: http://orcid.org/0000-0002-9073-9911; Gyuhee Kim: http://orcid.org/0000-0002-4054-979X; San gsoo Kim: http://orcid.org/0000-0001-9836-9823

\section{Authors' contribution}

\section{Conceptualization: SK}

Formal analysis: TRL, JMA, GK

Funding acquisition: SK

Writing - original draft: TRL, JMA, GK, SK

Writing - review \& editing: TRL, SK

\section{Acknowledgments}

This work was funded by a program (PJ01167402) from the RDA (Rural Development Administration) and a program (NRF-2012M3A9D1054705) from the NRF (National Research Foundation of Korea) and computationally supported by a program (NRF-2010-0018156, NTIS1711048528) from KISTI GSDC.

\section{Supplementary materials}

Supplementary data including three tables and 29 figures can be found with this article online at http://www. genominfo.org/src/sm/gni-15-178-s001.pdf.

\section{References}

1. van Dijk EL, Auger H, Jaszczyszyn Y, Thermes C. Ten years of next-generation sequencing technology. Trends Genet 2014; 30:418-426.

2. D'Agostino N, Tripodi P. NGS-based genotyping, high-throughput phenotyping and genome-wide association studies laid the foundations for next-generation breeding in horticultural crops. Diversity 2017;9:38.

3. Seemann T. Ten recommendations for creating usable bioinformatics command line software. Gigascience 2013;2:15.

4. Goecks J, Nekrutenko A, Taylor J; Galaxy Team. Galaxy: a comprehensive approach for supporting accessible, reproducible, and transparent computational research in the life sciences. Genome Biol 2010;11:R86.

5. Korean Bioinformation Center. Bioexpress. Daejeon: Korean Bioinformation Center, 2017. Accessed 2017 Nov 1. Available from: https:// bioexpress.kobic.re.kr.

6. R Core Team. R: a language and environment for statistical computing. Vienna: R Foundation for Statistical Computing, 2017.

7. Merkel D. Docker: lightweight Linux containers for consistent development and deployment. Linux J 2014;2014:2.

8. Chang W, Cheng J, Allaire JJ, Xie Y, McPherson J. shiny: Web Application Framework for R. R package version 1.0.5. The Comprehensive R Archive Network, 2017. Accessed 2017 Nov 1. Available from: https://CRAN.R-project.org/package= shiny.

9. Danecek P, Auton A, Abecasis G, Albers CA, Banks E, DePristo MA, et al. The variant call format and VCFtools. Bioinformatics 2011;27:2156-2158.

10. Purcell S, Neale B, Todd-Brown K, Thomas L, Ferreira MA, Bender D, et al. PLINK: a tool set for whole-genome association and population-based linkage analyses. Am J Hum Genet 2007;81:559-575.

11. Buels R, Yao E, Diesh CM, Hayes RD, Munoz-Torres M, Helt $G$, et al. JBrowse: a dynamic web platform for genome visualization and analysis. Genome Biol 2016;17:66.

12. Trapnell C, Williams BA, Pertea G, Mortazavi A, Kwan G, van Baren MJ, et al. Transcript assembly and quantification by 
RNA-Seq reveals unannotated transcripts and isoform switching during cell differentiation. Nat Biotechnol 2010; 28:511-515.

13. Li H, Handsaker B, Wysoker A, Fennell T, Ruan J, Homer N, et al. The Sequence Alignment/Map format and SAMtools. Bioinformatics 2009;25:2078-2079.

14. Anders S, Pyl PT, Huber W. HTSeq: a Python framework to work with high-throughput sequencing data. Bioinformatics 2015;31:166-169.

15. Robinson MD, McCarthy DJ, Smyth GK. edgeR: a Bioconductor package for differential expression analysis of digital gene expression data. Bioinformatics 2010;26:139-140.

16. Young MD, Wakefield MJ, Smyth GK, Oshlack A. Gene ontol- ogy analysis for RNA-seq: accounting for selection bias. Genome Biol 2010;11:R14.

17. Li Y, Andrade J. DEApp: an interactive web interface for differential expression analysis of next generation sequence data. Source Code Biol Med 2017;12:2.

18. Nelson JW, Sklenar J, Barnes AP, Minnier J. The START App: a web-based RNAseq analysis and visualization resource. Bioinformatics 2017;33:447-449.

19. Cuellar-Partida G, Renteria ME, MacGregor S. LocusTrack: Integrated visualization of GWAS results and genomic annotation. Source Code Biol Med 2015;10:1.

20. Ziegler GR, Hartsock RH, Baxter I. Zbrowse: an interactive GWAS results browser. PeerJ Comput Sci 2015;1:e3. 


\title{
SUPPLEMENTARY INFORMATION
}

IVAG: An Integrative Visualization Application for Various Types of Genomic Data Based on R-Shiny and the Docker Platform

\author{
Tae-Rim Lee ${ }^{\S}$, Jin Mo Ahn ${ }^{\S}$, Gyuhee $\mathrm{Kim}^{\S}$, Sangsoo Kim*
}

Department of Bioinformatics and Life Science, Soongsil University, Seoul 06978, Korea 
Supplementary Table 1. R packages used in IVAG

\begin{tabular}{|c|c|}
\hline Name & Reference \\
\hline shiny & $\begin{array}{l}\text { Chang W, Cheng J, Allaire JJ, Xie Y, McPherson J (2017). shiny: Web } \\
\text { Application Framework for R. R package version 1.0.5. https://CRAN.R- } \\
\text { project.org/package=shiny }\end{array}$ \\
\hline shinydashboard & $\begin{array}{l}\text { Chang W, Ribeiro BB (2017). shinydashboard: Create Dashboards with 'Shiny'. R } \\
\text { package version 0.6.1. https://CRAN.Rproject.org/package=shinydashboard }\end{array}$ \\
\hline shinycssloaders & $\begin{array}{l}\text { Sali A (2017). shinycssloaders: add CSS loading animations to 'shiny' outputs. R } \\
\text { package version 0.2.0. https://CRAN.R-project.org/package=shinycssloaders }\end{array}$ \\
\hline shinyjs & $\begin{array}{l}\text { Attali D (2017). shinyjs: easily improve the user experience of your shiny apps in } \\
\text { seconds. R package version 0.9.1. https://CRAN.R-project.org/package=shinyjs }\end{array}$ \\
\hline RColorBrewer & $\begin{array}{l}\text { Neuwirth E (2014). RColorBrewer: ColorBrewer Palettes. R package version 1.1- } \\
\text { 2. https://CRAN.R-project.org/package=RColorBrewer }\end{array}$ \\
\hline gplots & $\begin{array}{l}\text { Warnes GR, Bolker B, Bonebakker L, Gentleman R, Liaw WH, Lumley T, } \\
\text { Maechler M, Magnusson A, Moeller S, Schwartz M, Venables B (2016). } \\
\text { gplots: various R programming tools for plotting data. R package version 3.0.1. } \\
\text { https://CRAN.R-project.org/package=gplots }\end{array}$ \\
\hline ggplot2 & $\begin{array}{l}\text { Wickham H. ggplot2: elegant graphics for data analysis. Springer-Verlag, New } \\
\text { York, } 2009\end{array}$ \\
\hline easyGgplot2 & $\begin{array}{l}\text { Kassambara A (2014). easyGgplot2: perform and customize easily a plot with } \\
\text { ggplot2. R package version 1.0.0.9000. http://www.sthda.com }\end{array}$ \\
\hline rmarkdown & $\begin{array}{l}\text { Allaire JJ, Cheng J, Xie Y, McPherson J, Chang W, Allen J, Wickham H, Atkins } \\
\text { A, Hyndman R, Arslan R (2017). rmarkdown: dynamic documents for R. R } \\
\text { package version 1.6. https://CRAN.R-project.org/package=rmarkdown }\end{array}$ \\
\hline rtracklayer & $\begin{array}{l}\text { Lawrence M, Gentleman R, Carey V. "rtracklayer: an }\{\mathrm{R}\} \text { package for interfacing } \\
\text { with genome browsers". Bioinformatics 2009;25:1841-1842. }\end{array}$ \\
\hline DT & $\begin{array}{l}\text { Xie Y (2016). DT: a wrapper of the JavaScript library 'DataTables'. R package } \\
\text { version 0.2. https://CRAN.R-project.org/package=DT }\end{array}$ \\
\hline data.table & $\begin{array}{l}\text { Dowle M, Srinivasan A (2017). data.table: extension of `data.frame`. R package } \\
\text { version 1.10.4. https://CRAN.R-project.org/package=data.table }\end{array}$ \\
\hline gridExtra & $\begin{array}{l}\text { Auguie B (2017). gridExtra: miscellaneous functions for "Grid" graphics. R } \\
\text { package version 2.3. https://CRAN.R-project.org/package=gridExtra }\end{array}$ \\
\hline GenomicFeatures & $\begin{array}{l}\text { Lawrence M, Huber W, Pagés H, Aboyoun P, Carlson M, Gentleman R, et al. } \\
\text { Software for computing and annotating genomic ranges. PLoS Comput Biol } \\
\text { 2013;9:e1003118 }\end{array}$ \\
\hline edgeR & $\begin{array}{l}\text { Robinson MD, McCarthy DJ, Smyth GK. edgeR: a bioconductor package for } \\
\text { differential expression analysis of digital gene expression data. Bioinformatics } \\
\text { 2010;26:139-140 }\end{array}$ \\
\hline goseq & $\begin{array}{l}\text { Young MD, Wakefield MJ, Smyth GK, Oshlack A. Gene ontology analysis for } \\
\text { RNA-seq: accounting for selection bias. Genome Biol 2010;11:R14 }\end{array}$ \\
\hline pheatmap & $\begin{array}{l}\text { Kolde R (2015). pheatmap: Pretty Heatmaps. R package version 1.0.8. } \\
\text { https://CRAN.R-project.org/package=pheatmap }\end{array}$ \\
\hline plotly & $\begin{array}{l}\text { Sievert C, Parmer C, Hocking T, Chamberlain S, Ram K, Corvellec M, Despouy P } \\
\text { (2017). plotly: create interactive web graphics via 'plotly.js'. R package version } \\
\text { 4.7.1. https://CRAN.R-project.org/package=plotly }\end{array}$ \\
\hline manhattanly & $\begin{array}{l}\text { Bhatnagar S (2016). manhattanly: interactive Q-Q and Manhattan plots using } \\
\text { 'plotly.js'. R package version 0.2.0. https://CRAN.R- } \\
\text { project.org/package=manhattanly }\end{array}$ \\
\hline LDheatmap & $\begin{array}{l}\text { Shin JH, Blay S, McNeney B, Graham J (2006). LDheatmap: an R function for } \\
\text { graphical display of pairwise linkage disequilibria between single nucleotide } \\
\text { polymorphisms. J Stat Softw 2006;16:Code Snippet } 3\end{array}$ \\
\hline GWASTools & $\begin{array}{l}\text { Gogarten SM, Bhangale T, Conomos MP, Laurie CA, McHugh CP, Painter I, et } \\
\text { al. GWASTools: an R/Bioconductor package for quality control and analysis of }\end{array}$ \\
\hline
\end{tabular}


genetics

org.At.tair.db genome-wide association studies. Bioinformatics 2012;28:3329-3331

Warnes G, with contributions from Gregor Gorjanc, Leisch F, Man M (2013). Genetics: population genetics. R package version 1.3.8.1.

https://CRAN.R-project.org/package=genetics

Carlson M (2015). org.At.tair.db: Genome wide annotation for Arabidopsis. R package version 3.2.3. 
Supplementary Table 2. Plugins attached in JBrowse

\begin{tabular}{ll}
\hline Name & Reference \\
\hline gccontent & https://github.com/elsiklab/gccontent \\
narrowpeak & https://github.com/cmdcolin/narrowpeak \\
gwasviewer & https://github.com/elsiklab/gwasviewer \\
bedGraph to BigWig & https://github.com/ENCODE-DCC/kentUtils \\
\hline
\end{tabular}


Supplementary Table 3. Example data source

\begin{tabular}{ll}
\hline Name & Reference \\
\hline RNA-seq single factor & https://www.ebi.ac.uk/arrayexpress/experiments/E-MTAB-4298/ \\
RNA-seq multiple factor & https://www.ebi.ac.uk/arrayexpress/experiments/E-MTAB-4243/ \\
GWAS and LD analysis & $\begin{array}{l}\text { Tutorial data of R package GAPIT } \\
\text { http://zzlab.net/GAPIT/GAPIT Tutorial Data.zip }\end{array}$ \\
& \begin{tabular}{l} 
numeric to vcf format conversion was carried out using TASSEL \\
\hline
\end{tabular} \\
\hline
\end{tabular}


<Supplementary material 2>

\section{Index}

1. RNA-Seq

01. DEG analysis for single factor $\ldots \ldots \ldots 3$

02. DEG Visualization ............. 04

03. Gene Ontology Enrichment Analysis …06 04. DEG analysis for multiple factor ..... 09 05. DEG visualization \& Gene Ontology Enrichment for pre-analyzed data $\ldots \ldots \ldots \ldots 1$

06. Input files $\ldots \ldots \ldots \ldots \ldots \ldots$

2. GWAS

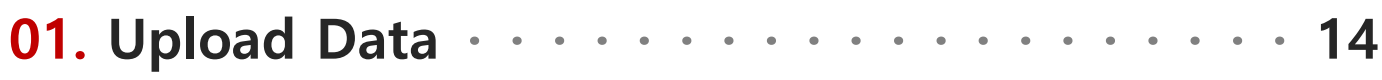

02. Manhattan and QQ Plot $\ldots \ldots 15$

03. LD heatmap Visualization $\ldots \ldots \ldots$

04. Input files $\ldots \ldots \ldots \ldots \ldots \ldots$ 
3. Genome Browser

01. Docker installation $\ldots \ldots \ldots . . . .20$

02. Launching Docker Image $\ldots \ldots 20$

03. Upload Data - Build ......... 21

04. Upload Data - Upload $\ldots \ldots \ldots 22$

05. Upload Data - Build_folder $\ldots \ldots \ldots 23$

06. Remove Data - Track ......... 23

07. Remove Data - Dataset ........ 24

08. RNA-Seq \& GWAS $\ldots \ldots \ldots . . . . .24$

09. RNA-Seq $\ldots \ldots \ldots \ldots . \ldots . \ldots 24$

10. GWAS $\ldots \ldots \ldots \ldots . \ldots 26$ 


\section{$<1.1$ DEG analysis for single factor $>$}

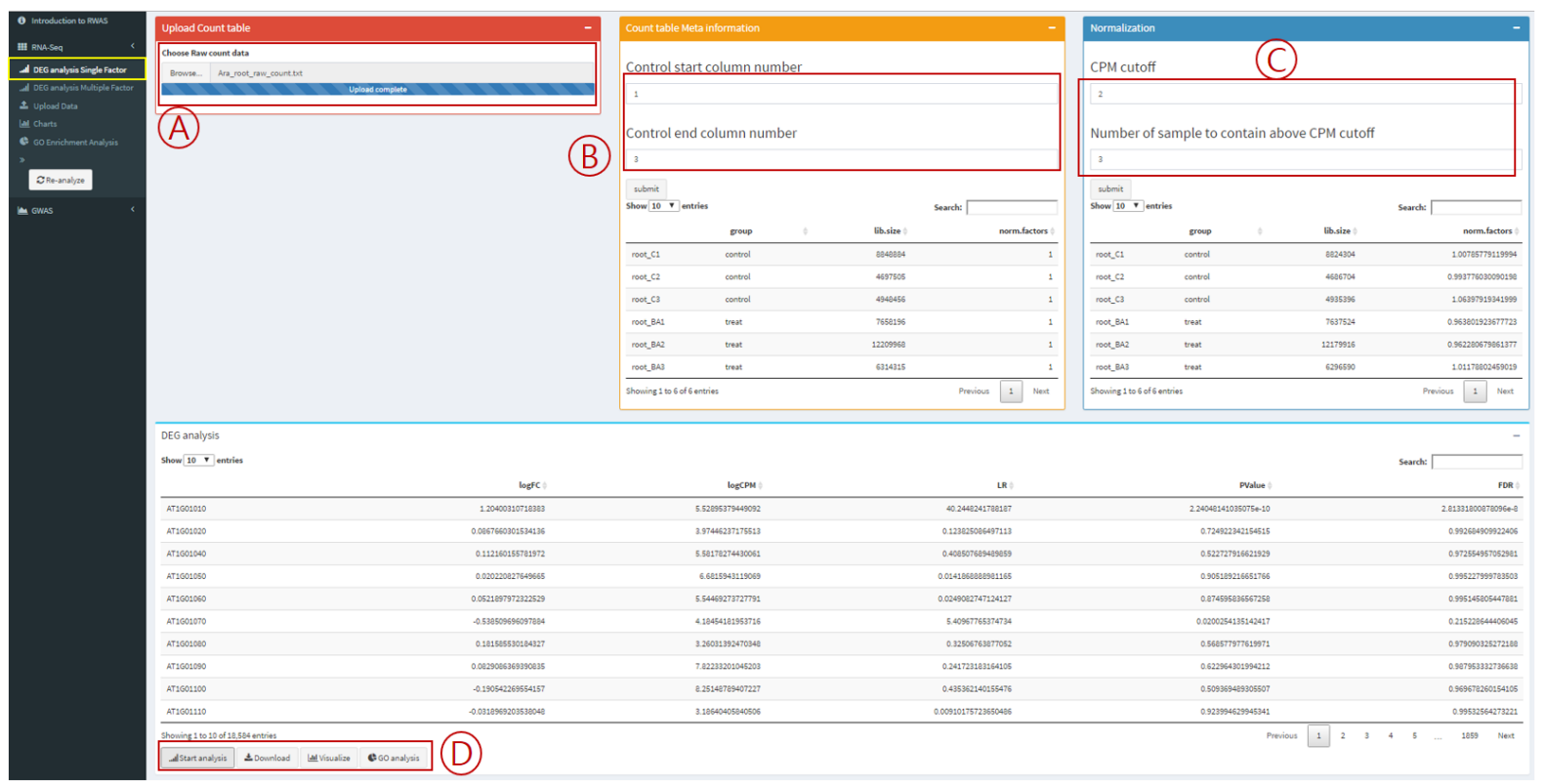

<Figure 1. DEG single factor analysis page >

(A): Upload count table generated using htseq-count or similar software after mapping RNA-Seq data to reference Genome.

(B): Specify the range of control columns.

(C): In order to analyze differentially expressed genes, each genes need at least $6 \sim 10$ counts. However, in raw count table, there are lots of genes with 0 counts which need to be filtered out. We use count per million to filter out genes with low or no counts. CPM cutoff criteria filters gene out using the smallest library size. For example, if the smallest library size was 4,000,000, CPM cutoff 2 would filter out genes with less than 8 counts. Number of samples to contain above CPM cutoff criteria can be specified as to how many samples must satisfy above filtering criteria. For example, raw count table with samples having 3 replicates each, if we specify Number of sample to contain above CPM as 3, at least 3 samples must meet the filtering criteria in order to proceed.

(D): Clicking the Start analysis button will run DEG analysis and produce the results as a table. Users can click Download button to get the results as text file format. Visualization button will lead the users to a page where they can generate Heatmap, Volcano plot, PCA analysis plot with the DEG analysis result. Clicking the $\mathrm{GO}$ analysis button will lead to a page where Gene Ontology Enrichment analysis can be done. 


\section{$<1.2$ DEG Visualization $>$}
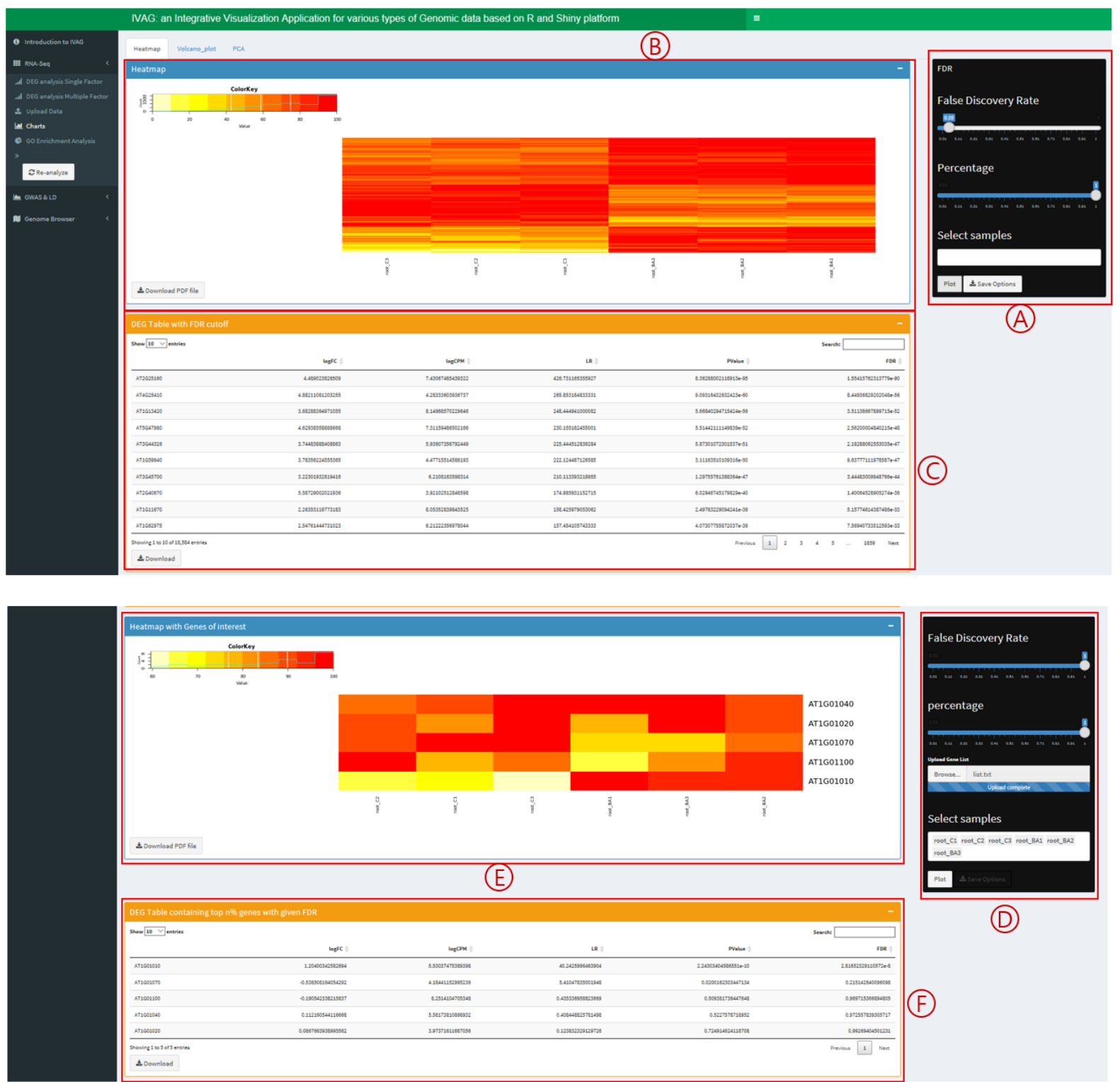

(E)
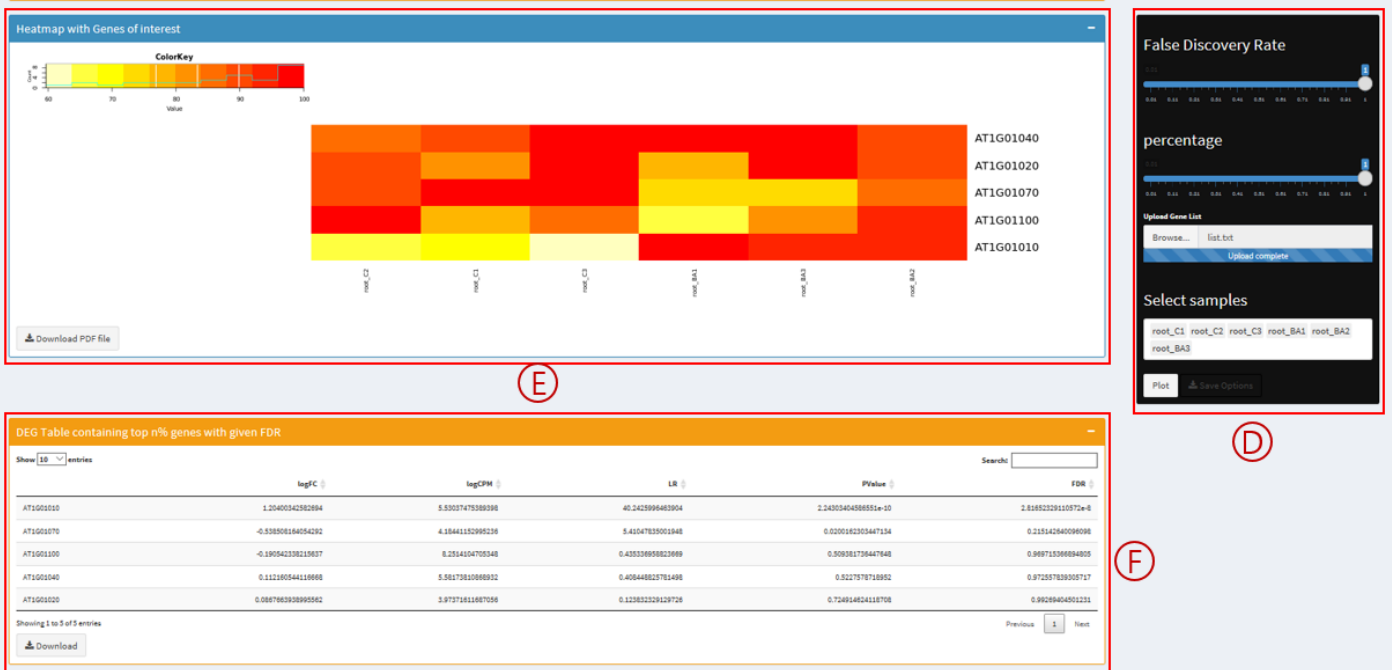

(D)

<Figure 2. Heatmap visualization>

(A): Specify False Discovery Rate to filter out genes to be used to draw heatmap. Percentage can be set to draw heatmap with top $n \%$ of filtered genes. User can specify samples to be drawn on heatmap.

(B): Generated heatmap 
(C): Shows DEG analysis table of genes used to create heatmap. Click Download button to download table in text file format.

(D): Specify False Discovery Rate to filter out genes to be used to draw heatmap. Percentage can be set to draw heatmap with top $n \%$ of filtered genes. User can specify samples to be drawn on heatmap. Gene list can be uploaded to draw heatmap with genes of interest.

(E): Generated heatmap.

(F): Shows DEG analysis table of genes used to create heatmap. Click Download button to download table in text file format.

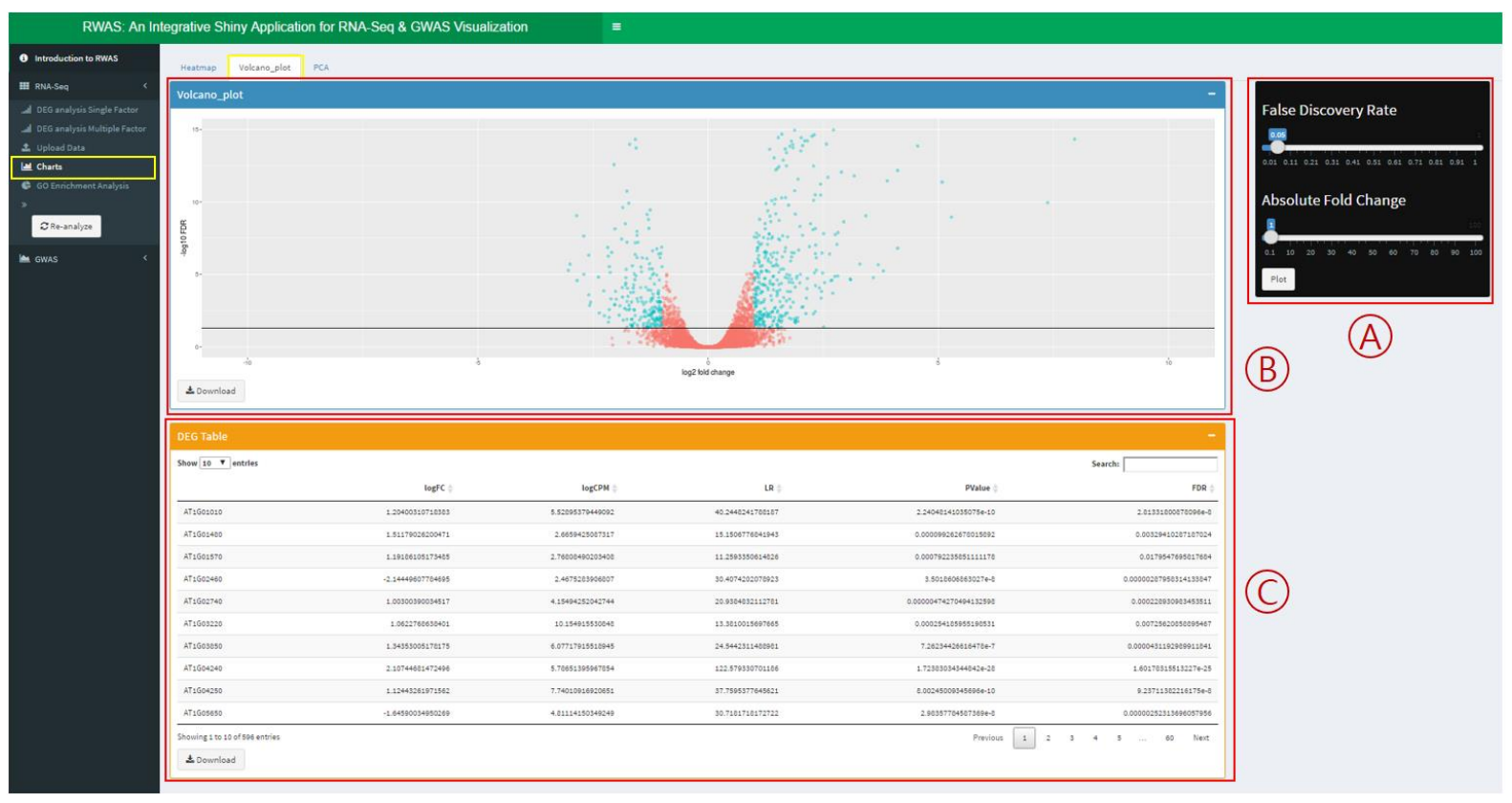

$<$ Figure 3. Volcano plot visualization >

(A): User can specify False Discovery Rate or Absolute Fold Change to generate Volcano plot.

(B): Generates volcano plot by plotting logf fold change versus - $\log 10$ (False Discovery Rate). User can download generated plot in PDF format by clicking the Download button.

(C): DEG analysis result table for genes used to plot volcano plot is shown. This table can be downloaded in text format by clicking the Download button. 


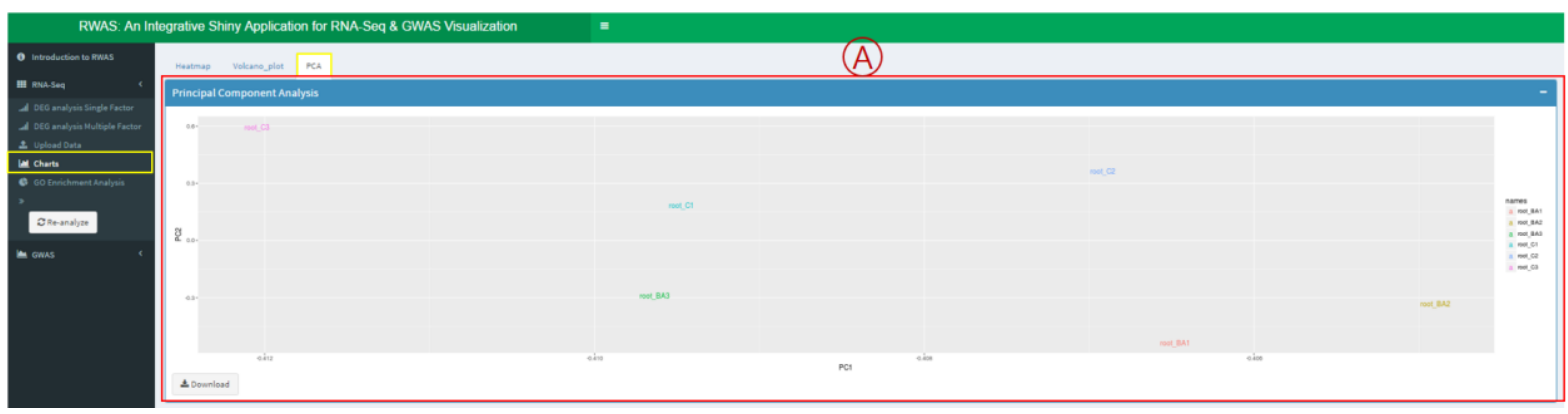

$<$ Figure 4. PCA plot visualization>

(A): Generates Principal analysis plot. This can be downloaded in PDF format by clicking the Download button.

\section{$<1.3$ Gene Ontology Enrichment Analysis >}
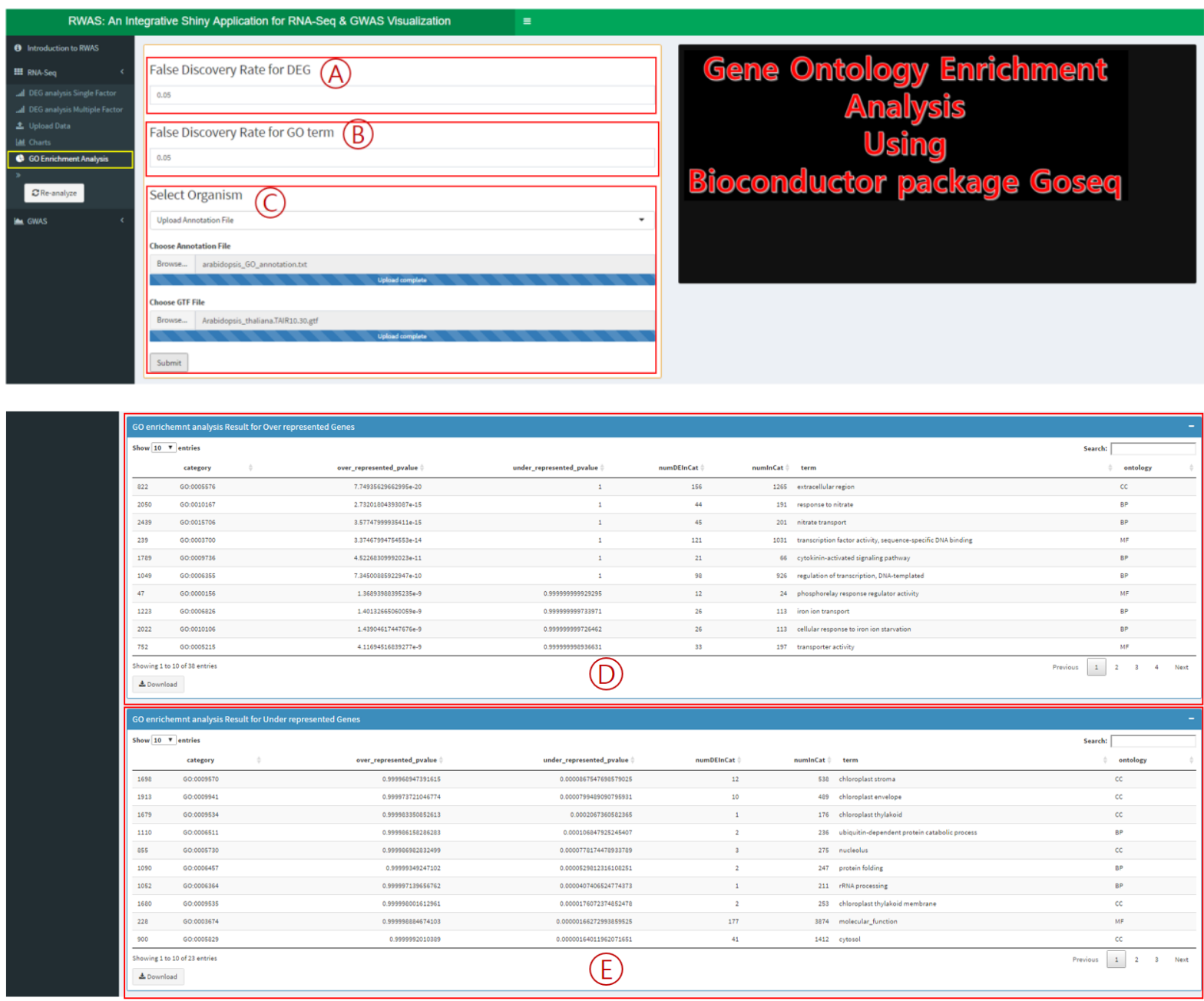

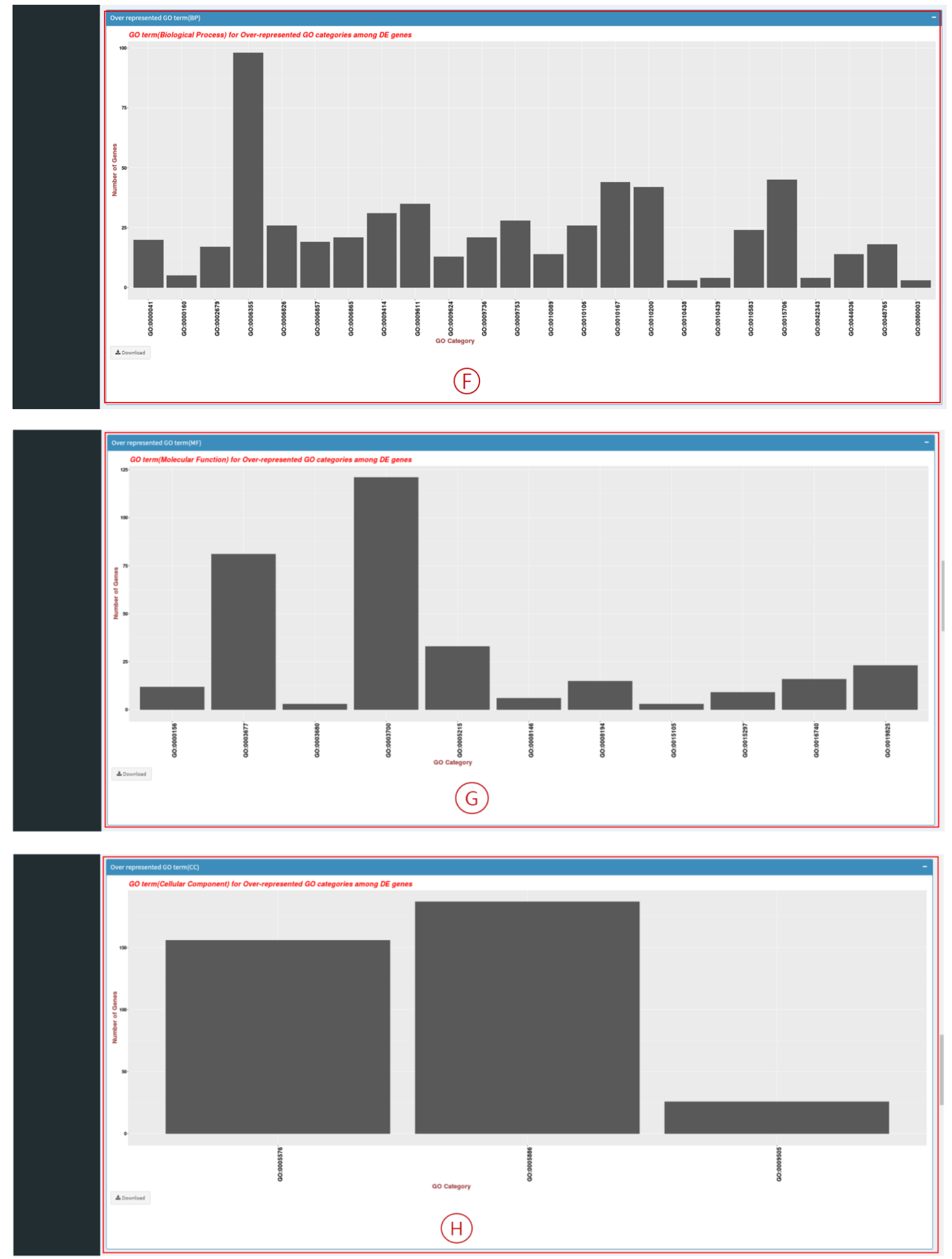

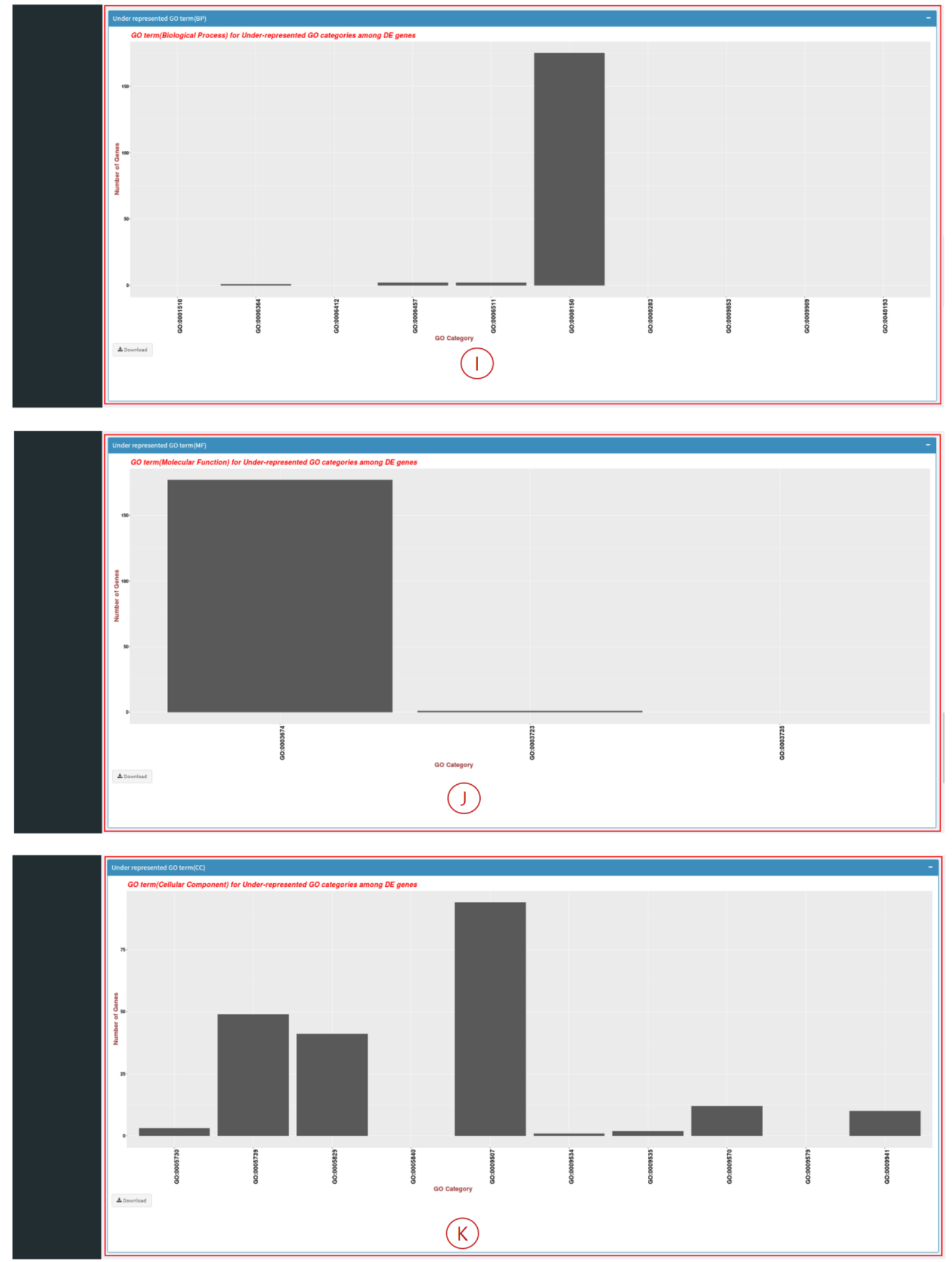

<Figure 5. Gene Ontology Enrichment analysis page>

(A): Specify filtering criteria(False Discovery Rate) for differentially expressed genes which will go through Gene Ontology Enrichment analysis. 
(B): Specify False Discovery Rate to be used in Gene Ontology Enrichment analysis.

(C): Upload Gene Ontology annotation file and GTF file.

(D): Shows over represented Gene Ontology Enrichment analysis result among differentially expressed genes. $5^{\text {th }}$ column(numlnCat) shows how many genes are allocated to the specific category and $4^{\text {th }}$ column(numDEInCat) shows among those genes, how many of them are differentially expressed. Users can click Download button to retrieve the results in text file format.

(E): Shows under represented Gene Ontology Enrichment analysis result among differentially expressed genes. $5^{\text {th }}$ column(numlnCat) shows how many genes are allocated to the specific category and $4^{\text {th }}$ column(numDElnCat) shows among those genes, how many of them are differentially expressed. Users can click Download button to retrieve the results in text file format.

$(\odot) \mathbb{B}$ : Shows the histogram of differentially expressed genes with specific Gene Ontology term and ontology such as biological process, cellular component and molecular function. These graphs can be downloaded in PDF format by clicking the Download button.

\section{<1.4 DEG analysis for multiple factor $>$}

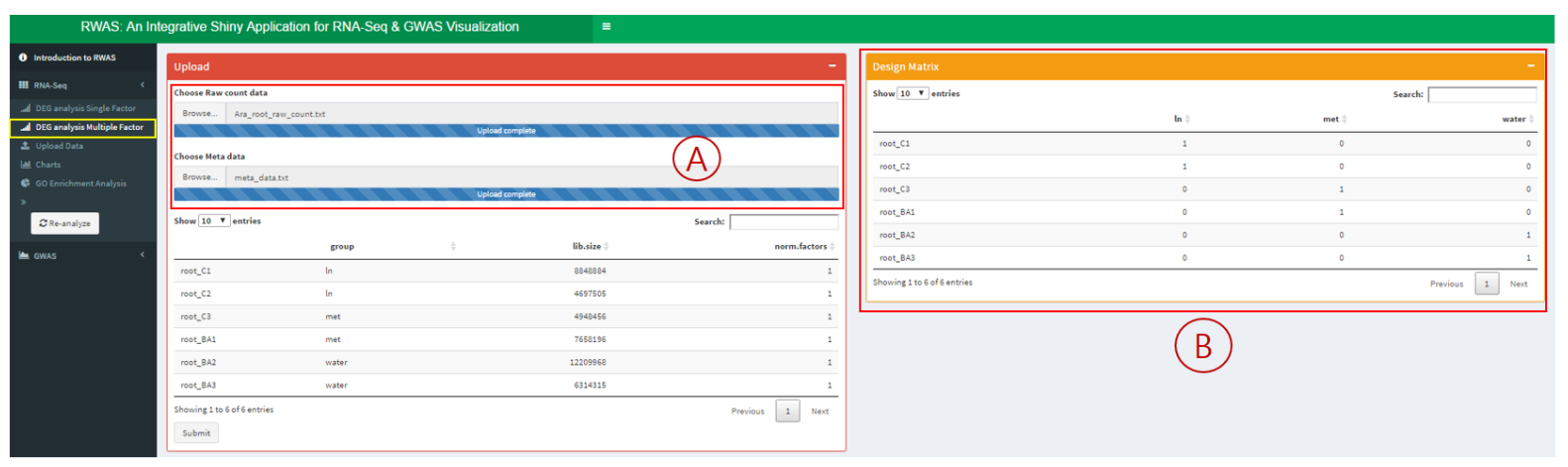



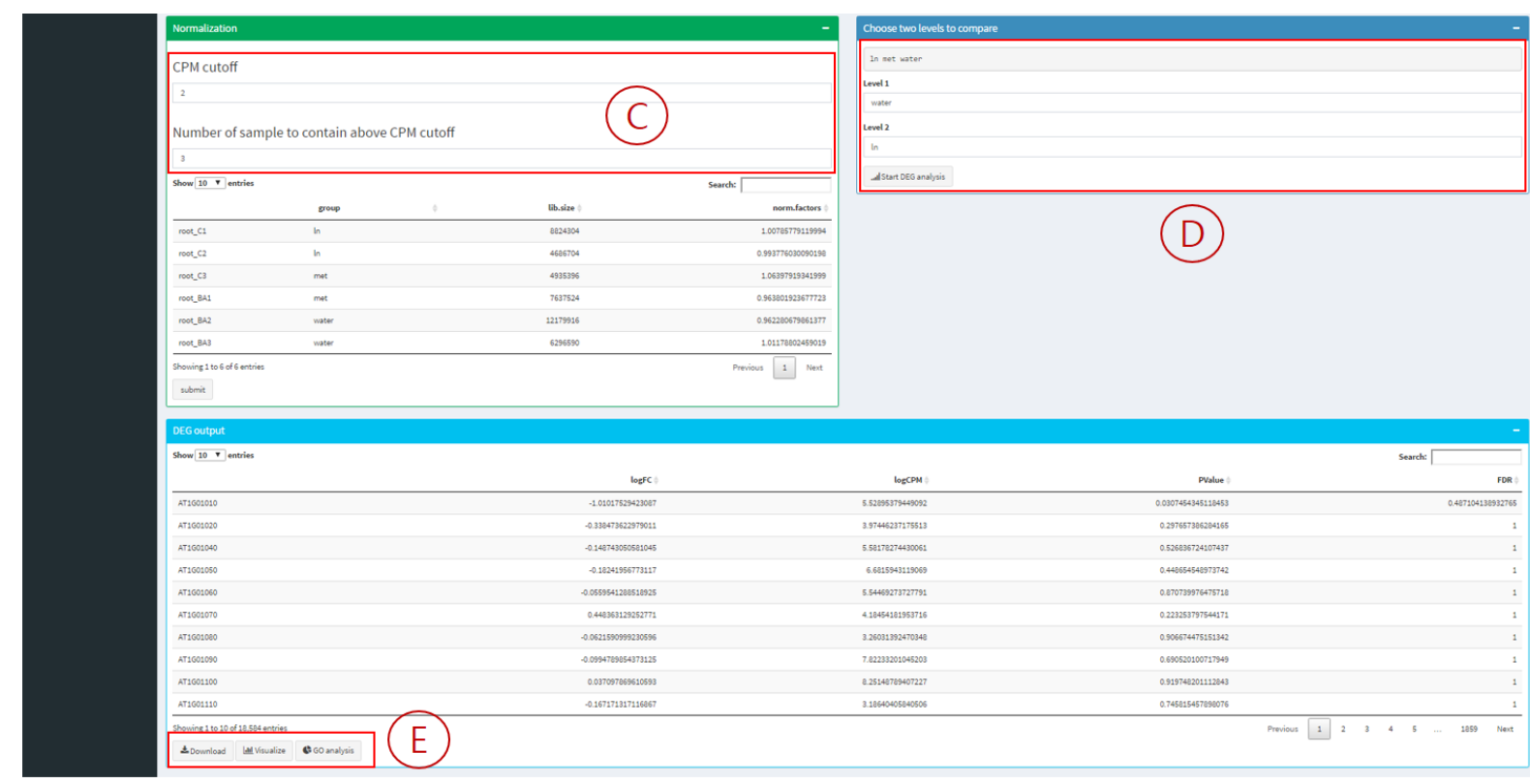

<Figure 6. Multi factor DEG analysis multi factor page>

(A): Upload count table generated using htseq-count or similar software after mapping RNA-Seq data to reference Genome with meta information file.

(B): Generates design matrix when all the files are uploaded.

(C): In order to analyze differentially expressed genes, each genes need at least $6 \sim 10$ counts. However, in raw count table, there are lots of genes with 0 counts which need to be filtered out. We use count per million to filter out genes with low or no counts. CPM cutoff criteria filters gene out using the smallest library size. For example, if the smallest library size was 4,000,000, CPM cutoff 2 would filter out genes with less than 8 counts. Number of samples to contain above CPM cutoff criteria can be specified as to how many samples must satisfy above filtering criteria. For example, raw count table with samples having 3 replicates each, if we specify Number of sample to contain above CPM as 3, at least 3 samples must meet the filtering criteria in order to proceed.

(D): Among multiple samples, choose two samples to proceed DEG analysis.

(E): Clicking the Start analysis button will run DEG analysis and produce the results as a table. Users can click Download button to get the results as text file format. Visualization button will lead the users to a page where they can generate Heatmap, Volcano plot, PCA analysis plot with the DEG analysis result. Clicking the GO analysis button will lead to a page where Gene Ontology Enrichment analysis can be done. 


\section{<1.5 DEG visualization and Gene Ontology Enrichment analysis for pre-analyzed data>}

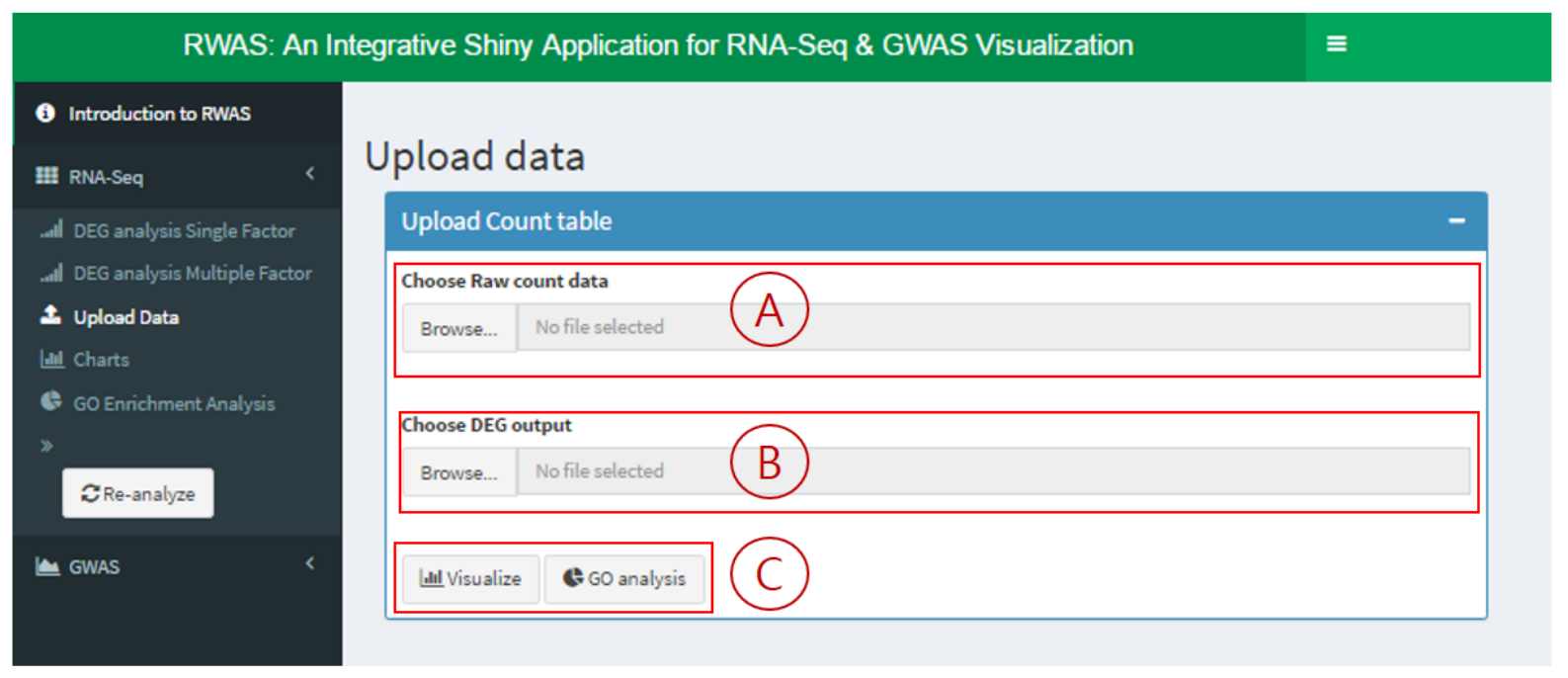

<Figure 7. Pre-analyzed data upload page>

(A): Upload count table generated using htseq-count or similar software after mapping RNA-Seq data to reference Genome.

(B): Upload DEG analysis result table.

(C): Visualization button will lead the users to a page where they can generate Heatmap, Volcano plot, PCA analysis plot with the DEG analysis result. Clicking the GO analysis button will lead to a page where Gene Ontology Enrichment analysis can be done. 


\section{$<1.6$ Input files $>$}

In1 In2 meth1 meth2 water1 water2

ATIG01010 $440 \quad 366 \quad 419625 \quad 334 \quad 482$

$\begin{array}{lllllll}\text { AT1G01020 } & 1095 & 1030 & 856 & 1447 & 759 & 1183\end{array}$

AT1901030 000030140

$\begin{array}{lllllll}\text { AT1G01040 } & 2455 & 1706 & 2672 & 4204 & 1834 & 309\end{array}$

AT1G01046

$\begin{array}{llllll}11 & 10 & 20 & 52 & 17 & 30\end{array}$

$4204 \quad 1834$

3098

AT1G01050

$\begin{array}{lllll}11180 & 11030 & 10013 & 16152 & 8180\end{array}$

11682

AT1G01060 $652 \quad 473 \quad 800 \quad 1410 \quad 642 \quad 996$

$\begin{array}{lllllll}\text { ATIG01070 } & 109 & 97 & 91 & 119 & 58 & 113\end{array}$

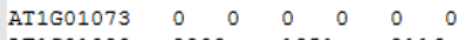

AT1G01080

$2209 \quad 1951 \quad 2116$

AT1G01090

$8647 \quad 8519 \quad 7271$

3061

$10956 \quad 1655$

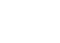

2 in

AT1G01100

15938

1394210564

AT1G01110 000000000

$\begin{array}{lllllll}\text { AT1G01115 } & 0 & 0 & 0 & 0 & 0 & 0\end{array}$

$\begin{array}{lllllll}\text { AT1G01120 } & 8 & 1 & 15 & 17 & 6 & 19 \\ \text { AT1G01130 } & 68 & 65 & 51 & 62 & 43 & 60\end{array}$

$\begin{array}{lllllll}\text { ATIG01140 } & 478 & 463 & 624 & 1003 & 529 & 829\end{array}$

aT1601140

AT1G01150

AT1G01160

AT1G01170

$\begin{array}{lllll}478 & 463 \quad 624 & 1003 & 529\end{array}$

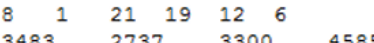

$\begin{array}{llllll}3483 & 2737 & 3300 & 4585 & 2468 & 3396\end{array}$

$\begin{array}{lllllll}80 & 5 & 3 & 15 & 34 & 20 & 19\end{array}$

$\begin{array}{lllllll}\text { AT1G01183 } & 0 & 0 & 0 & 0 & 0 & 0\end{array}$

AT1G01190

AT1G01200

AT1G01210

AT1G01220

AT1G01225

AT1G01230

AT 1 G01240

AT1G01250

AT1G01260

AT1G01270

$\begin{array}{llllll}26 & 24 & 11 & 5 & 2 & 8\end{array}$

$\begin{array}{lllll}604 \quad 628 & 539 & 898 & 441 \quad 645\end{array}$

$\begin{array}{llllll}3277 & 2761 & 4580 & 7004 & 3652 & 5094\end{array}$

$\begin{array}{llllll}779 & 686 & 810 & 1294 & 754 & 1085\end{array}$

$\begin{array}{llll}2199 & 2120 & 1979 & 2881\end{array}$

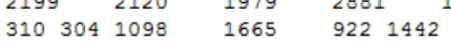

$\begin{array}{llll}2 & 0 & 0 & 1\end{array}$

0

$\begin{array}{lllll}1340 & 1136 & 1258 \\ 0 & 0 & 0 & 0 & 0\end{array}$

$1943 \quad 1035$

2318

00

A

"logFC" "logCPM" "LR" "PValue" "FDR"

"AT1G01010" 1.19688737736099 $5.52688808335964 \quad 39.3089106372121 \quad 3.61786523882999 \mathrm{e}-10 \quad 4.80222720383886 \mathrm{e}-08$ $\begin{array}{llllll}\text { "AT1G01020" } 0.0822804977656206 & 3.97165716089378 & 0.111434656713485 & 0.738516674183035 & 0.992950029705138\end{array}$ "AT1G01030" $-0.162468882715308 \quad 0.0823715495057947 \quad 0.0610995702734676 \quad 0.804766390210944 \quad 0.994580412175897$ "ATIG01040" $0.106377858075599 \quad 5.57910426454308 \quad 0.37042230935759 \quad 0.5427741633203390 .974600223816041$ "AT1G01050" $0.0153574773648363 \quad 6.67932177116587 \quad 0.00814645735631103 \quad 0.92808241163436 \quad 0.995983980196374$ $\begin{array}{llllllll}\text { "AT1G01060" } 0.0456180046924779 & 5.54156373113906 & 0.019399476207294 & 0.889227311214195 & 0.995931204225289\end{array}$ $\begin{array}{lllllll}\text { "AT1G01070" } & -0.544012070552485 & 4.18302675004016 & 5.48539400852262 & 0.0191759975507977 & 0.211398693538396\end{array}$ $\begin{array}{lllllllll}\text { "AT1G01080" } 0.174667523566418 & 3.26203485385487 & 0.299539868880617 & 0.584171025511136 & 0.978544418926473\end{array}$ "AT1G01090" $0.0770064556487513 \quad 7.820012190007720 .2062350769147590 .649734453606224 \quad 0.987057549001829$ "ATIG01100" -0.194374421491345 8.249997826352350 .4509221210632430 .5018973787755290 .967135588725176 "AT1G01110" $-0.0356166922910766 \quad 3.18109685561797 \quad 0.011510637873462 \quad 0.914560789141767 \quad 0.995983980196374$ $\begin{array}{lllll}\text { "AT1G01120" } 0.641291331519332 & 6.3387621639768 & 11.0961455503092 \quad 0.000865073303584205 \quad 0.0196921958851816\end{array}$ $\begin{array}{llllllll}\text { "AT1G01130" } 0.467864553000374 & 1.76456938585317 & 0.977622941476459 & 0.322786369058292 & 0.904971916676201\end{array}$ "AT1G01140" $-0.542815616073306 \quad 2.63788851959664 \quad 2.13563384343845 \quad 0.1439109667218810 .68944588214333$ "AT1G01160" $-0.0296144956218807 \quad 5.15484537653471 \quad 0.0203745510116882 \quad 0.88649595793983 \quad 0.995931204225289$ $\begin{array}{llllllll}\text { "AT1G01170" } 0.0959514365512992 & 3.64734295039377 & 0.15817628576344 & 0.69084114621376 & 0.987813981559841\end{array}$ $\begin{array}{lllllll}\text { "AT1G01180" } 0.24596413317094 & 3.7881988269758 & 0.650354637870404 & 0.419985915804817 & 0.950470048855293\end{array}$ "AT1G01190" $-0.375672462290263 \quad 3.86317903951983 \quad 0.635708343635596 \quad 0.4252691542938290 .951393075845317$ $\begin{array}{llllllll}\text { "AT1G01200" } 0.246507464818891 & 3.01178043499523 & 0.779877708445121 & 0.377178543497332 & 0.933754390534284\end{array}$ 


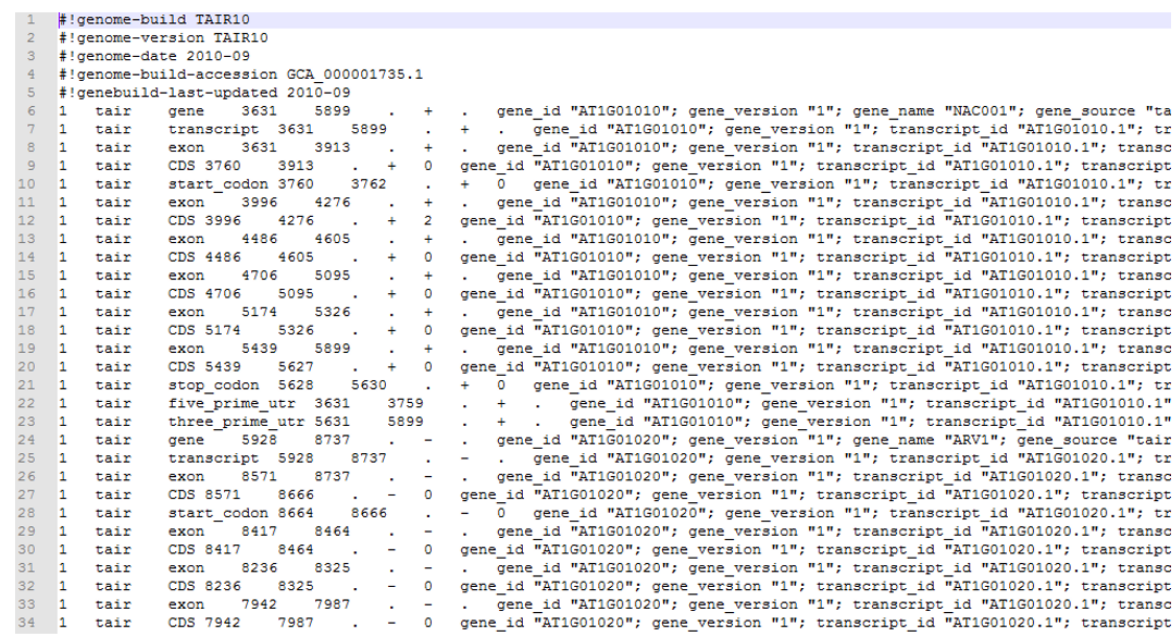

(D)

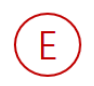

$<$ Figure 8. >

(A): Raw count table / Used in DEG analysis

(B): Meta information specifying which column belongs to which group in raw count table / Used in DEG analysis multiple factor.

(C): DEG analysis result / pre analyzed DEG result table can be uploaded. Column header must be the same as above figure but, 'logCPM' and 'LR' can be omitted.

(D): Gene Ontology annotation file / Text file containing Gene ID and corresponding Gene Ontology category. Gene ID must be the same as the ones used in raw count table.

(E): GTF file / Used in Gene Ontology Enrichment analysis to calculate gene length. 


\section{GWAS \& LD heatmap}

\section{$<2.1$ Upload Data >}

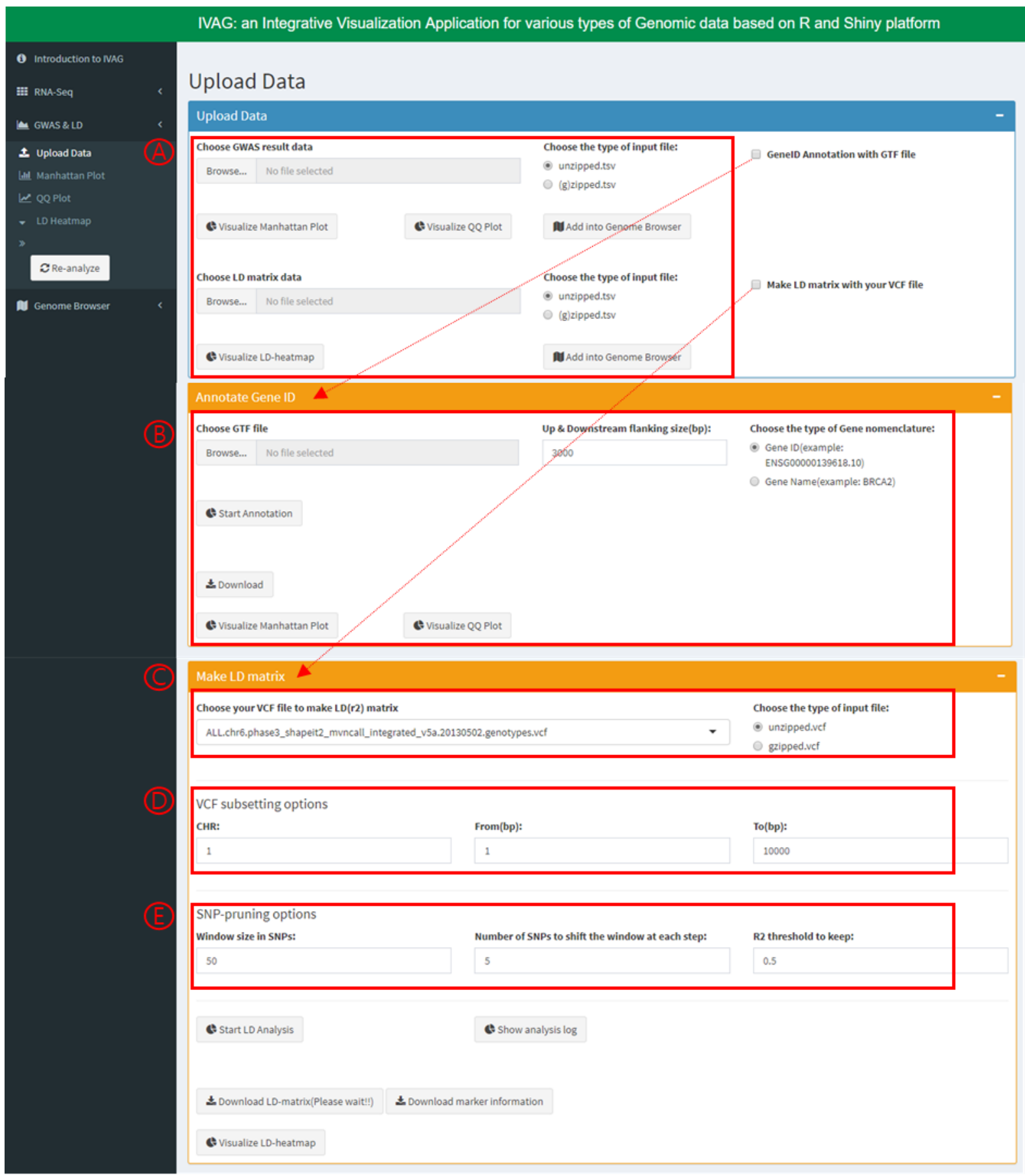

<Fig. 9. GWAS \& LD Upload and Analysis page>

(A): GWAS result and LD matrix files (TSV format) can be uploaded. Users can move into plotting page by clicking Visualize buttons. Also, uploaded files can be added into pre-built genome browser. 
(B): Users can annotate gene information into all markers in the GWAS result file.

(C): Choose a VCF file you want to use in the LD analysis.

(D): Subset the region of your interest by assigning CHR, Start, and END parameters.

(): All markers will be pruned using PLINK analysis option "--indep-pairwise" to reduce the number of markers to be used in LD calculation. Detailed information for this analysis can be found at http://zzz.bwh.harvard.edu/plink/summary.shtml\#prune.

\section{<2.2 Manhattan and QQ Plot Visualization >}

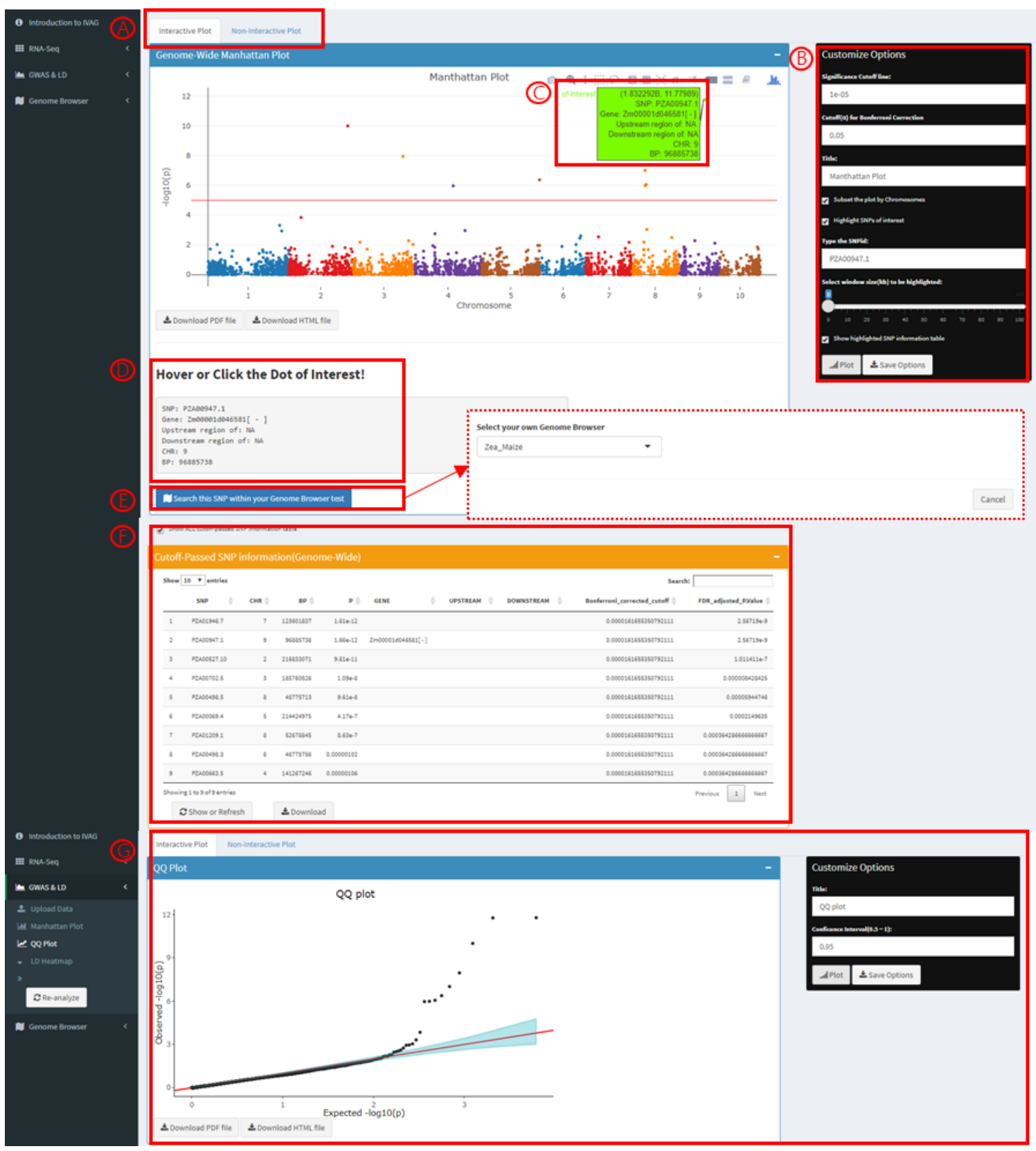

<Fig. 10. Manhattan and QQ plot visualization page> 
(A): Users can select what type of plot to draw; Interactive or Non-Interactive.

(B): Adjust specific parameters to customize your plot. You can subset the plot by chromosome and highlight the SNPs of your interest.

(C): Detailed information will be shown when the cursor is hovered over a specific point of interest.

(D): If you click a specific dot, all information for that dot will be recoded here.

(E): User can move directly into the genome browser to search the SNP selected the previous step. To use this function, genome browser construction should be done in advance.

(F): Information of all markers that passed the cutoff will be shown here.

(a): QQ plot can also be drawn. Light blue shade indicates the confidence interval for the null hypothesis that assumes there is no association between SNP and a trait.

\section{<2.3 LD heatmap Visualization >}

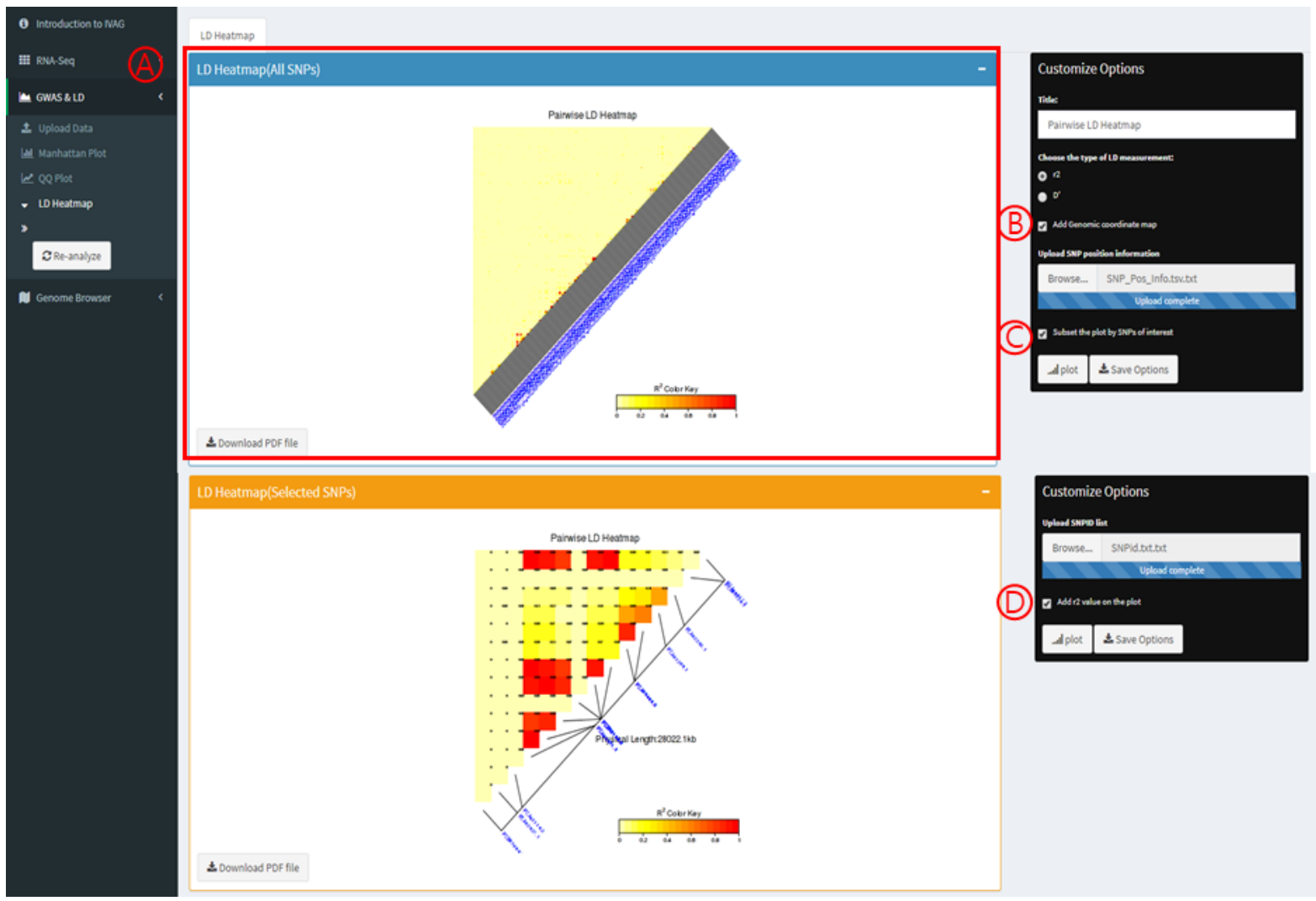

<Fig. 12. LD heatmap visualization page>

(A): All markers in the LD matrix (uploaded or generated) will be plotted on the upper panel.

(B): Genomic coordinate map can be added when genomic coordinate information is given.

(C): The LD heatmap can be subset by uploading a list of markers of your interest 
(D): $r 2$ value for each SNP-SNP pair can be added on the plot.

\section{$<2.4$ Input Files $>$}

\section{GWAS}

\begin{tabular}{|l|r|r|r|}
\hline SNP & Chromosome & Position & P.value \\
\hline PZA01946.7 & 7 & 123601837 & $1.61 \mathrm{E}-12$ \\
\hline PZA00947.1 & 9 & 96885738 & $1.66 \mathrm{E}-12$ \\
\hline PZA00527.10 & 2 & 216833071 & $9.81 \mathrm{E}-11$ \\
\hline PZA00702.5 & 3 & 185760526 & $1.09 \mathrm{E}-08$ \\
\hline PZA00498.5 & 8 & 48775713 & $9.61 \mathrm{E}-08$ \\
\hline PZA00069.4 & 5 & 214424975 & $4.17 \mathrm{E}-07$ \\
\hline PZA01209.1 & 8 & 52678845 & $8.63 \mathrm{E}-07$ \\
\hline PZA00498.3 & 8 & 48775756 & $1.02 \mathrm{E}-06$ \\
\hline PZA00663.5 & 4 & 141267246 & $1.06 \mathrm{E}-06$ \\
\hline PZA02808.12 & 2 & 44606596 & 0.000143238 \\
\hline tb1.11 & 1 & 264848126 & 0.000483664 \\
\hline
\end{tabular}

<Fig. 13. GWAS_Result.tsv $-1>$

1. IVAG intakes GWAS result summary statistics file that comprise Marker ID, Chromosome, position, and $\mathrm{p}$-value columns in order.

\begin{tabular}{|c|c|c|c|c|c|c|}
\hline SNP & Chromosome & Position & P.value & GENE & UPSTREAM & DOWNSTREAM \\
\hline PZA01946.7 & 7 & 123601837 & $1.61 \mathrm{E}-12$ & NA & NA & NA \\
\hline PZA00947.1 & 9 & 96885738 & $1.66 \mathrm{E}-12$ & Zm00001d046581[ - ] & NA & NA \\
\hline PZA00527.10 & 2 & 216833071 & $9.81 \mathrm{E}-11$ & NA & NA & NA \\
\hline PZA00702.5 & 3 & 185760526 & $1.09 \mathrm{E}-08$ & NA & NA & NA \\
\hline PZA00498.5 & 8 & 48775713 & $9.61 \mathrm{E}-08$ & NA & NA & NA \\
\hline PZA00069.4 & 5 & 214424975 & 4.17E-07 & NA & NA & NA \\
\hline PZA01209.1 & 8 & 52678845 & 8.63E-07 & NA & NA & NA \\
\hline PZA00498.3 & 8 & 48775756 & $1.02 E-06$ & NA & NA & NA \\
\hline PZA00663.5 & 4 & 141267246 & $1.06 \mathrm{E}-06$ & NA & NA & NA \\
\hline PZA02808.12 & 2 & 44606596 & 0.000143238 & NA & NA & NA \\
\hline tb1.11 & 1 & 264848126 & 0.000483664 & NA & NA & NA \\
\hline
\end{tabular}

<Fig. 13. GWAS_Result.tsv - $2>$

2. Another version of the input file contains additional three columns which could be annotated from IVAG Gene ID annotation function. 


\section{LD heatmap -1}

\begin{tabular}{|c|c|c|c|c|c|c|c|c|c|c|c|c|c|c|c|c|}
\hline$\triangle$ & A & B & c & D & E & $F$ & & H & I & J & K & L & M & N & 0 & p \\
\hline 1 & & PZA02388.1 & PZA02174.2 & PZA03316.4 & PZA03316.1 & PZA03316.3 & (РZA00368.17) & PZA00368.11 & PZA00058.1 & PZA00058.5 & PZA00632.1 & PZA00416.4 & PZA00416.2 & PZA01601.1 & PZA03178.3 & PZA03178.1 \\
\hline 2 & PZA02388.1 & NA & 0.003131425 & 0.001322904 & $1.89 E-05$ & 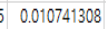 & 0.00236276 & $6.80 \mathrm{E}-05$ & 0.004041285 & 0.00030022 & 0.012297859 & 0.011686865 & $\begin{array}{ll}5 & 0.007863762\end{array}$ & $1.12 E-06$ & 60.004849854 & 40.001331938 \\
\hline 3 & PZA02174.2 & NA & NA & 0.0004783 & 0.021465195 & $\begin{array}{ll}5 & 0.001231729\end{array}$ & 0.00992238 & 0.024481744 & + 0.010871922 & 0.00101117 & 0.002377562 & 2. 0.016651214 & $4 \quad 0.029450244$ & 0.055942213 & B $\quad 0.005134748$ & $\begin{array}{ll}3 & 0.002694006\end{array}$ \\
\hline 4 & PZA03316.4 & NA & NA & NA & 0.06055998 & $8 \quad 0.003111189$ & $.71 \mathrm{E}-05$ & 4.94E- -05 & 0.000890745 & 0.007548125 & 0.002149237 & 0.005553662 & 20.000882867 & 0.006944157 & 0.000186331 & 0.001078003 \\
\hline 5 & PZA03316.1 & NA & NA & NA & NA & 0.031768088 & 0.00528988 & 0.004006694 & $4 \quad 0.030093192$ & 0.00026265 & 0.000316417 & 0.008766957 & $\begin{array}{ll}7 & 0.020765621\end{array}$ & 0.009565184 & $4 \quad 0.000657431$ & 0.015585438 \\
\hline 6( & & MA & nu & MA & nis & $\stackrel{M A}{\longrightarrow}$ & 0.025351076 & 0.000510142 & 0.014710731 & 0.007110129 & 0.00223847 & 0.000121821 & 10.000939886 & 0.002376762 & 0.000639039 & 90.001826396 \\
\hline 7 & PZAOOSOB.17 & NA & NA & NA & NA & NA & NA & 0.23464068 & 0.015698233 & 0.006223222 & 0.000859469 & 0.006282812 & $2 \quad 1.15 E-05$ & 0.002566236 & $5.83 \mathrm{E}-06$ & $1.23 \mathrm{E}-05$ \\
\hline 8 & PZA00368.1 & NA & NA & NA & NA & NA & NA & NA & 0.022382311 & 0.001951984 & 0.000375188 & $\begin{array}{ll}3 & 0.001289014\end{array}$ & $2.77 E-05$ & $8.82 E-08$ & B 0.000300585 & 50.005470765 \\
\hline 9 & PZA00058.1 & NA & NA & NA & NA & NA & NA & NA & NA & 0.079778481 & 0.0092099 & 0.010593625 & $5 \quad 0.017851142$ & 0.000487344 & $8.59 \mathrm{E}-05$ & 0.025033994 \\
\hline 10 & PZA00058.5 & NA & NA & NA & NA & NA & NA & NA & NA & NA & 0.007402666 & 0.029095655 & $\begin{array}{ll}5 & 0.010838279\end{array}$ & 0.003178828 & 8 0.003044483 & $8.38 E-05$ \\
\hline $11 \mathrm{P}$ & PZA00632.1 & NA & NA & NA & NA & NA & NA & NA & NA & NA & NA & 0.000169487 & 0.01006222 & 0.012087334 & $1.87 E-05$ & 0.001650081 \\
\hline $12 \mathrm{~F}$ & PZAO00416.4 & NA & NA & NA & NA & NA & NA & NA & NA & NA & NA & NA & 0.510533148 & 0.037531322 & 20.015276814 & $4 \quad 0.004631683$ \\
\hline $13 \mathrm{P}$ & PZA00416.2 & NA & NA & NA & NA & NA & NA & NA & NA & NA & NA & NA & NA & 0.028323356 & 60.012447245 & 0.000960156 \\
\hline $14 \mathrm{P}$ & PZA01601.1 & NA & NA & NA & NA & NA & NA & NA & NA & NA & NA & NA & NA & NA & 0.021422767 & 70.016685048 \\
\hline $15 \mathrm{P}$ & PZA03178.3 & NA & NA & NA & NA & NA & NA & NA & NA & NA & NA & NA & NA & NA & NA & 0.150240822 \\
\hline 16 P & PZA03178.1 & NA & NA & NA & NA & NA & NA & NA & NA & NA & NA & NA & NA & NA & NA & NA \\
\hline
\end{tabular}

<Fig. 14. LD_Matrix_Rsquare.tsv >

1. LD matrix file is tab-separated and has $r 2$ values for all SNP-SNP pairs in an upper triangular matrix form.

2. The first row (header) is SNPIDs sorted by their position.

3. The first column, colored in blue, should not be included in the input LD matrix file, but I just showed it to help you better understand this format. SNP IDs in column header and row header are ordered equally and you can interpret this LD matrix like r2 value between PZA00368.17 and PZA03316.3 is 0.025351076 as described in green.

4. Note that your input file SHOULD look like the red boxed one.

\section{LD heatmap - 2}

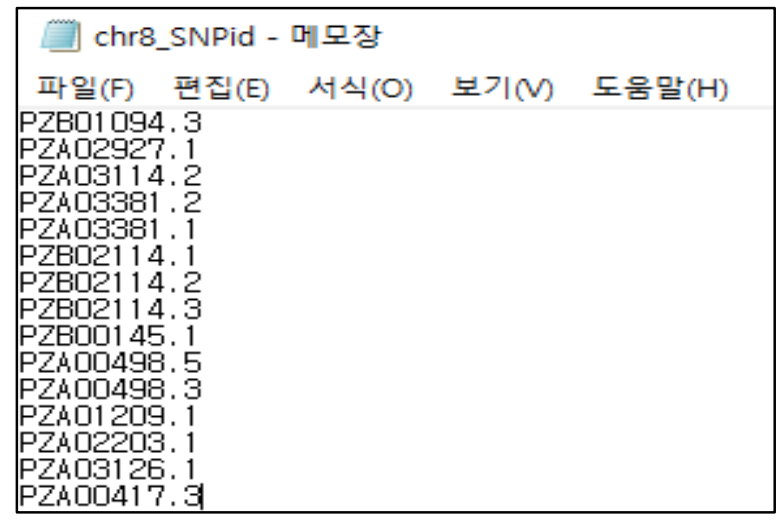

$<$ Fig. 15. SNPid.txt $>$

1. This text file has the list of SNP ids of your interest. You can extract a subset of LD heatmap that contains only SNPs specified with this file. 


\section{LD heatmap - 3}

\begin{tabular}{|c|l|r|r|}
\hline \multicolumn{1}{|c|}{ A } & \multicolumn{1}{c|}{ B } & \multicolumn{1}{c|}{ C } \\
\hline 1 & SNP & Chromosome & Position \\
\hline 2 & PZA02388.1 & 8 & 169137 \\
\hline 3 & PZA02174.2 & 8 & 4101256 \\
\hline 4 & PZA03316.4 & 8 & 4766593 \\
\hline 5 & PZA03316.1 & 8 & 4766694 \\
\hline 6 & PZA03316.3 & 8 & 4766801 \\
\hline 7 & PZA00368.17 & 8 & 5632196 \\
\hline 8 & PZA00368.1 & 8 & 5632308 \\
\hline 9 & PZA00058.1 & 8 & 5966657 \\
\hline 10 & PZA00058.5 & 8 & 5966698 \\
\hline 11 & PZA00632.1 & 8 & 6017018 \\
\hline 12 & PZA00416.4 & 8 & 8098163 \\
\hline 13 & PZA00416.2 & 8 & 8098271 \\
\hline 14 & PZA01601.1 & 8 & 8404207 \\
\hline 15 & PZA03178.3 & 8 & 11602192 \\
\hline
\end{tabular}

<Fig. 16. SNP_Pos_Info.tsv >

1. genomic coordinate information file requires three columns; SNP, Chromosome, and position. 


\section{JBrowse}

\section{$<3.1$ Docker installation >}

Install the docker before running IVAG. Docker is available on multiple platforms, on cloud and on-premises. Choose the best installation path for you and install it from the URL link below.

(https://docs.docker.com/engine/installation/)

\section{$<3.2$ Launching Docker Image >}

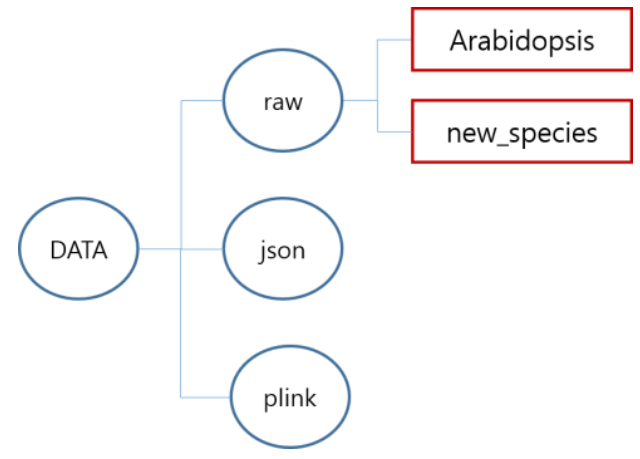

$<$ Figure 17. Example folder mounted on a docker >

1. Create DATA directory for mounting docker.

2. Create subdirectory raw, json, plink on Data directory.

- raw : Upload file for JBrowse

- json : Information about data uploaded on JBrowse

- plink : Save tmp, log files generated from IVAG LD analysis

3. Create subdirectory for each species under raw directory and put each data. (fasta, bam, gtf, gff3, bed, bw, vcf )

4. Specify DATA directory when launching docker image

\begin{tabular}{|c|l|}
\hline DATA directory & C:HUsers\#USER\#Desktop\#DATA \\
\hline Docker image & docker run -ti -v C:HUsers\#USER\#Desktop\#DATA:/jbrowse/my_data -p \\
launch commend & $8080: 80$-p 8383:3838 leetaerim/ivag:v1 /bin/bash -c "Rscript load.R" \\
\hline
\end{tabular}

$<$ Table 1. Example of launching docker image > 
$<3.3$ Upload Data - Build >

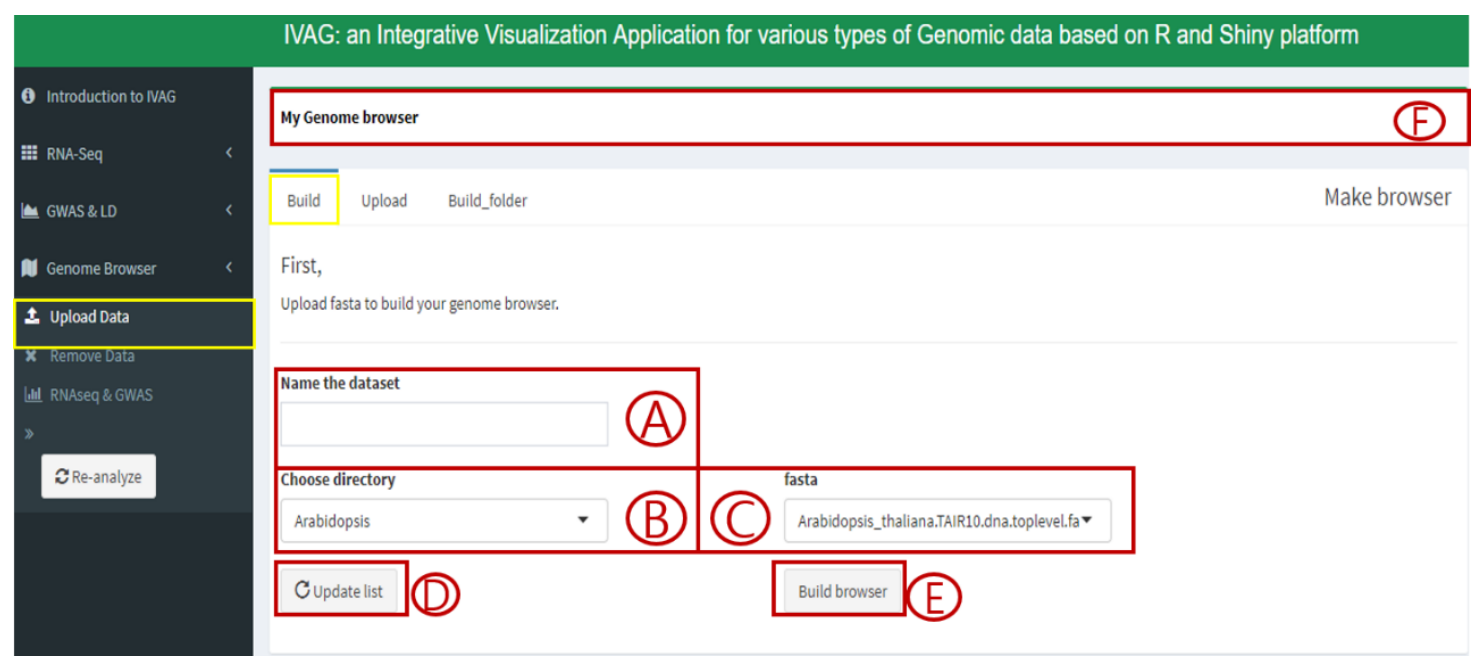

<Figure 18. Build Genome Browser page>

(A): Specify genome browser name

(B): Shows subdirectory of raw directory mounted on docker. Among the list, select subdirectory to construct genome browser.

(C): Shows files of subdirectory chosen from (B). Choose fasta file.

(D): Click Update list button to refresh list on (B).

(): Click Build browser button to construct genome browser.

(F): Leads to JBrowse page 
<3.4 Upload Data - Upload >

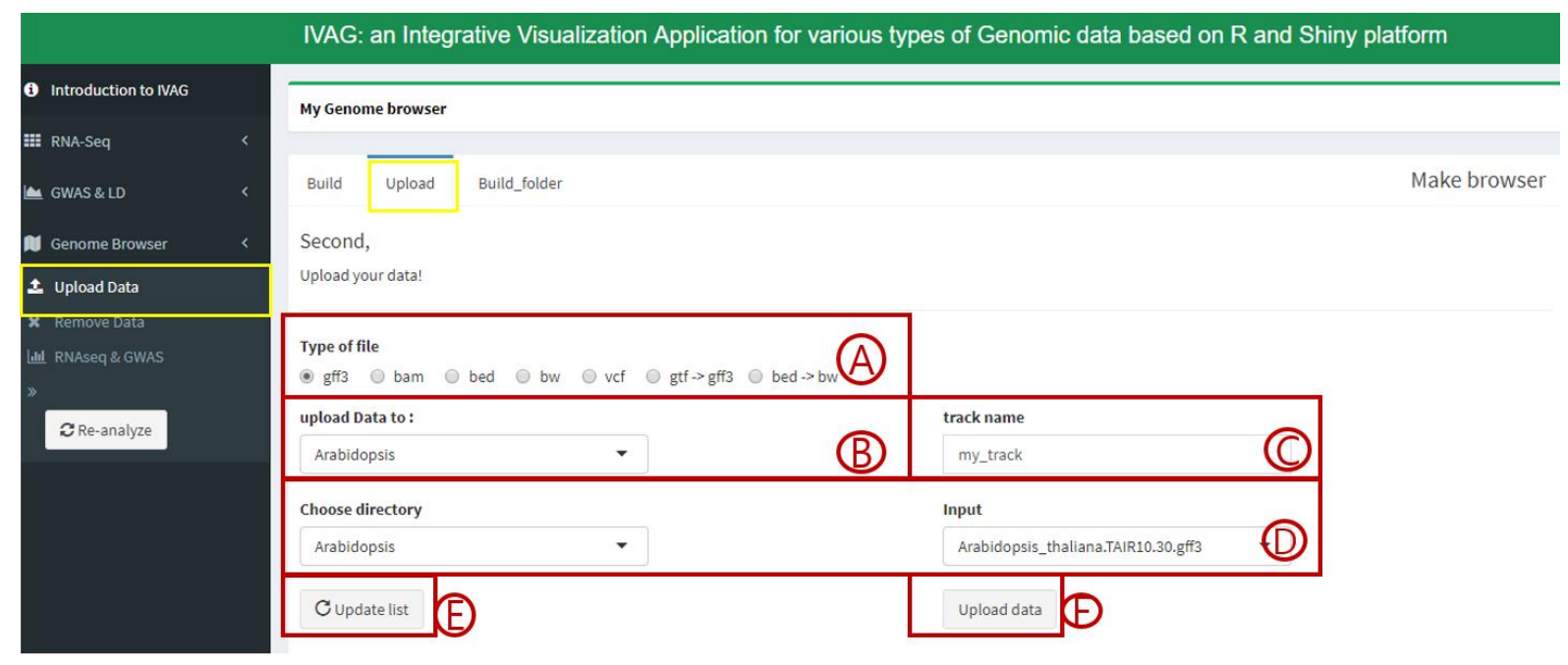

<Figure 19. Upload data to browser page>

(A): Specify file type to upload.

$\rightarrow$ vcf: Takes long time to index data for ID search. So asks whether user will index or not.

$\rightarrow$ bam : If bam file size is large, asks whether to divide file into pieces to upload.

(B): Select genome browser to upload.

(): Specify track name.

(D): Specify data to upload.

(E): Click Update list button to refresh list on (B), (D).

๑): Upload to genome browser. 
<3.5 Upload Data - Build_folder >

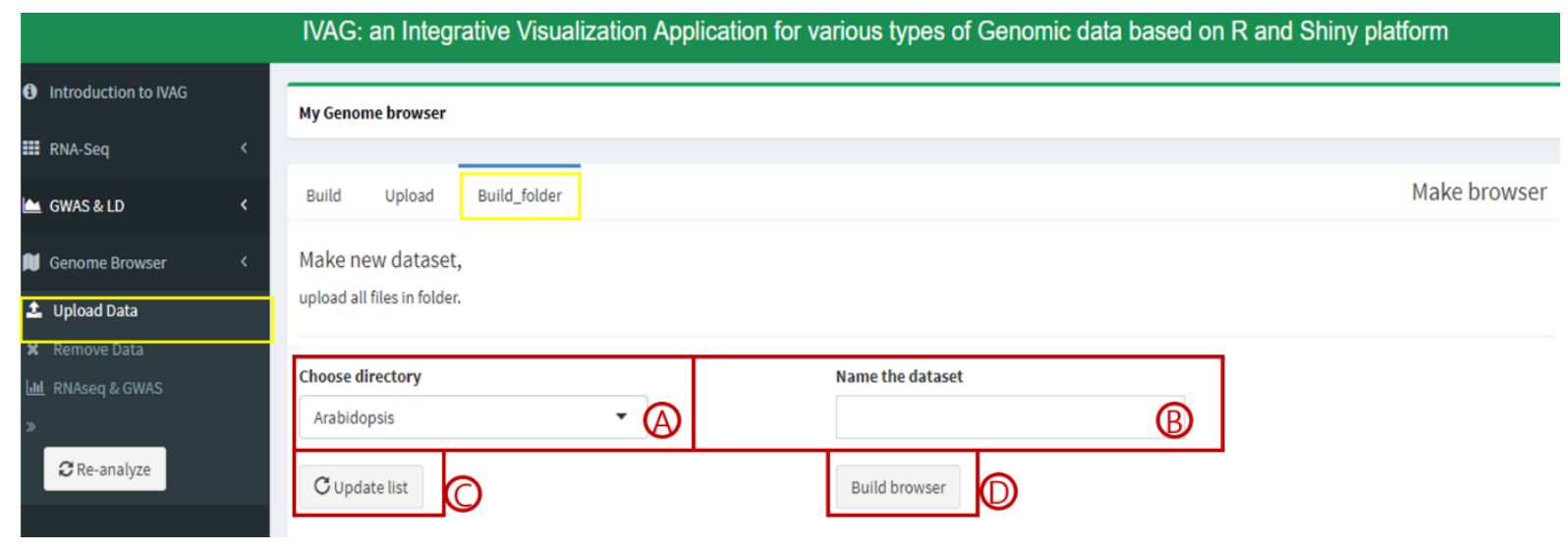

<Figure 20. Build and Upload folder to browser page>

(A): Build genome browser using fasta file in specified directory and upload all the file in the directory automatically.

(B): Specify genome browser name.

(C): Click Update list button to refresh list on (A)

(D): Build genome browser.

\section{<3.6 Remove Data - Track >}

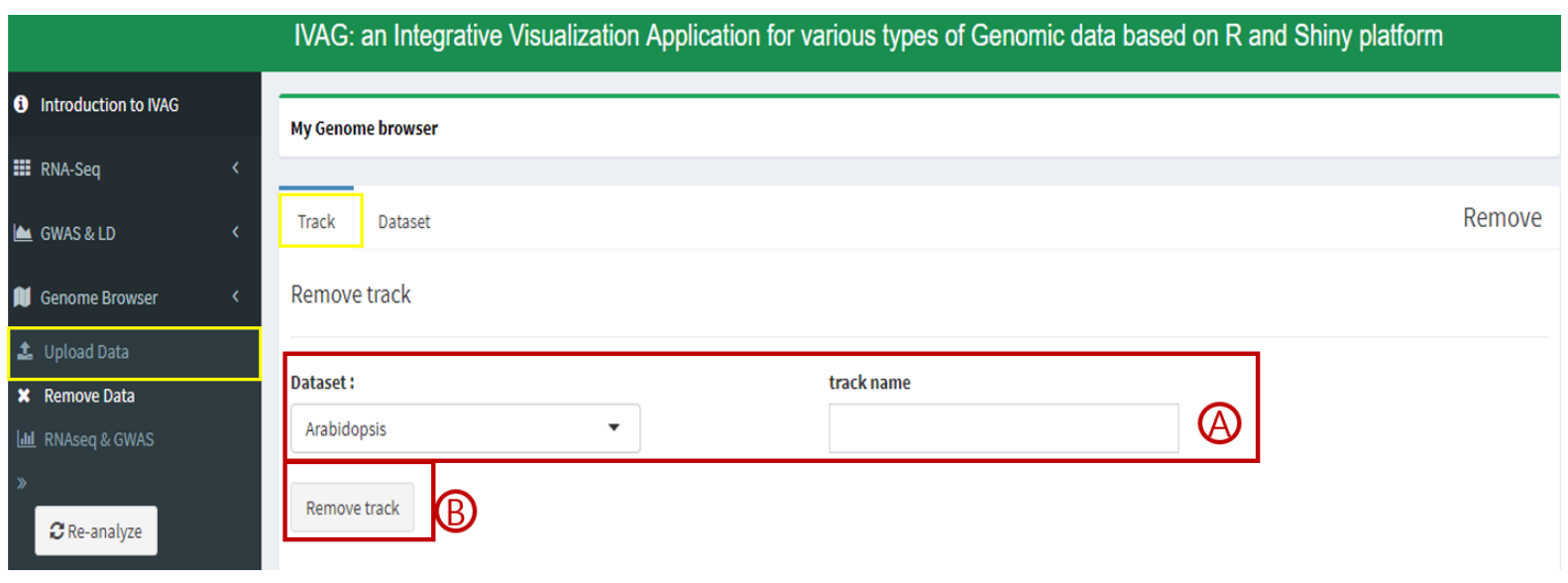

<Figure 21. Remove Data - Track page>

(A): Specify genome browser name and type track name to be deleted.

(B): Remove track. 


\section{$<3.7$ Remove Data - Dataset >}

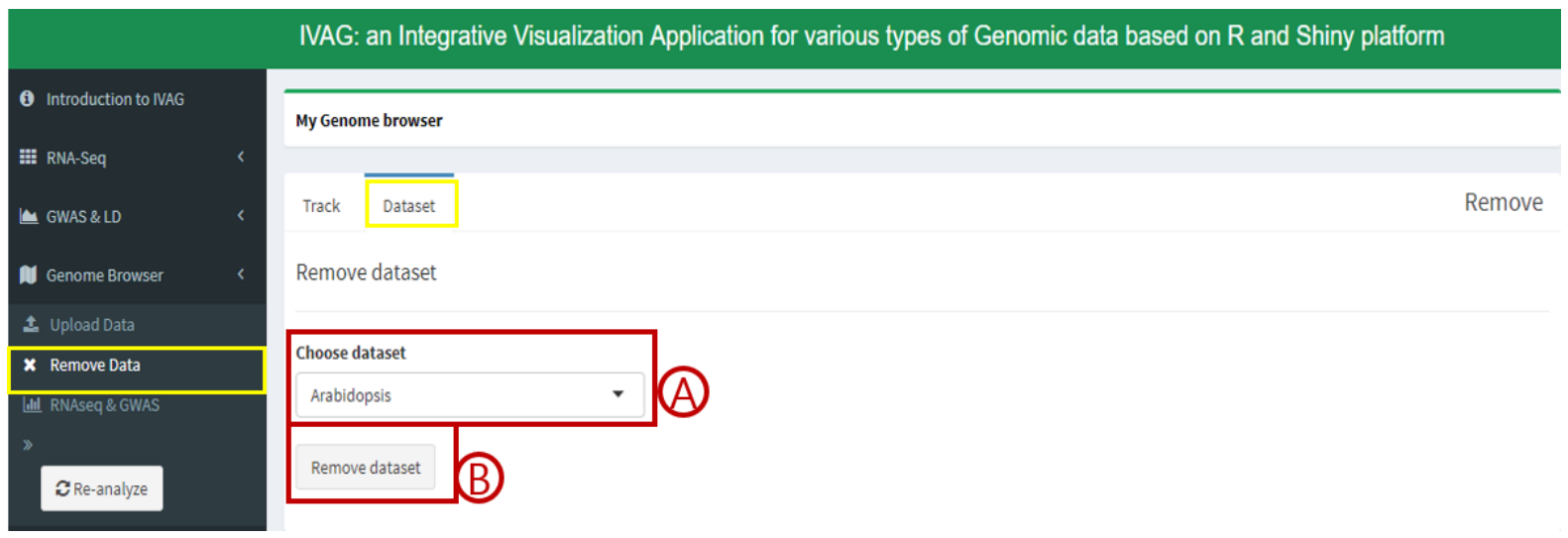

<Figure 22. Remove Data - Dataset page>

(A): Select genome browser to be removed.

(B): Remove genome browser.

\section{<3.8 RNA-Seq \& GWAS >}

IVAG RNA-Seq and GWAS analysis result can be uploaded to IVAG genome browser. Results can be uploaded via file but can be omitted by clicking "Add into Genome Browser" button on analysis page.

\section{<3.9 RNA-Seq \& GWAS - RNA-Seq >}

\begin{tabular}{|c|c|c|c|c|c|c|}
\hline Q6 my $=D E G$ & AT1G06870 & AT1G07135 & AT1G07400 & AT1G07630 & AT1G07870 & AT1G08315 \\
\hline AT1G06620 & & AT1G072 & & & & AT1G08320 \\
\hline & & & $1 \mathrm{G} 07350$ & & & \\
\hline
\end{tabular}

$<$ Figure 23. JBrowse RNAseq peak track >

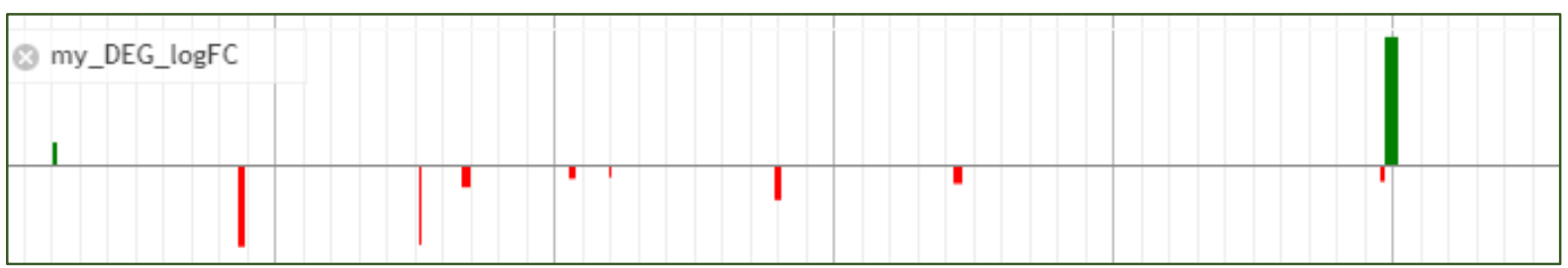

<Figure 24. JBrowse RNAseq peak with logFC track >

IVAG RNA-Seq analysis result can be uploaded in 3 track types.

(1) peak : DEG list is uploaded on JBrowse in bed file format (Figure 23)

(2) peak with $\log F C$ : Uploaded to JBrowse in BigWig format. (Figure 24) 
(3) peak with logCPM : Uploaded to JBrowse in BigWig format.

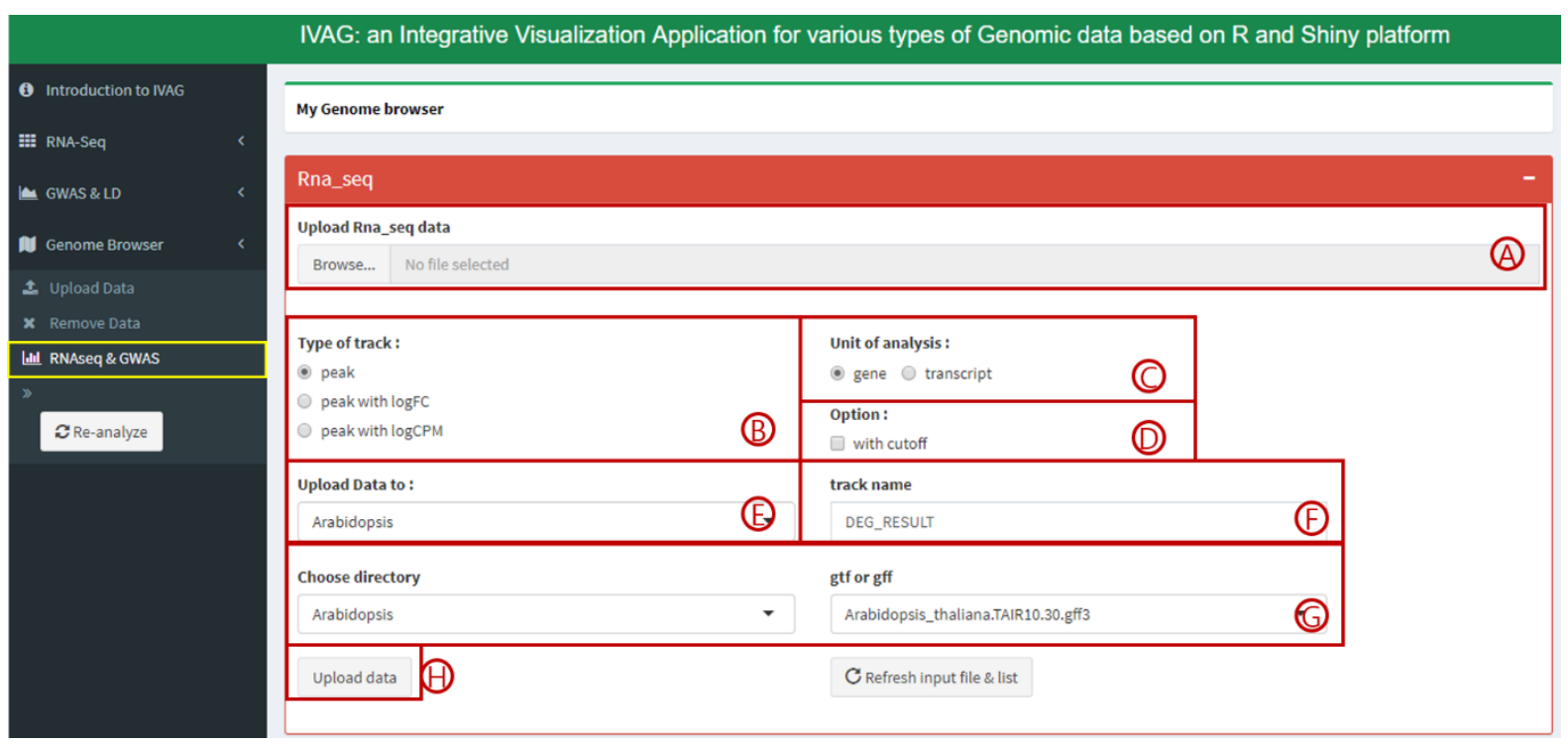

<Figure 25. RNAseq\&GWAS - RNAseq >

(A): Upload DEG analysis result table.

(B): Specify track type.

(C): Specify analysis level(gene, transcript ).

(D): Can specify FDR cutoff for DEG.

(E): Select genome browser to be uploaded.

$\odot$ : Set track name.

(G): Genome browser needs gene positions in order to process DEG list. Therefore GTF file needs to be specified.

$\oplus$ (A): Create RNA-Seq track. 


\section{$<3.10$ RNA-Seq \& GWAS - GWAS >}

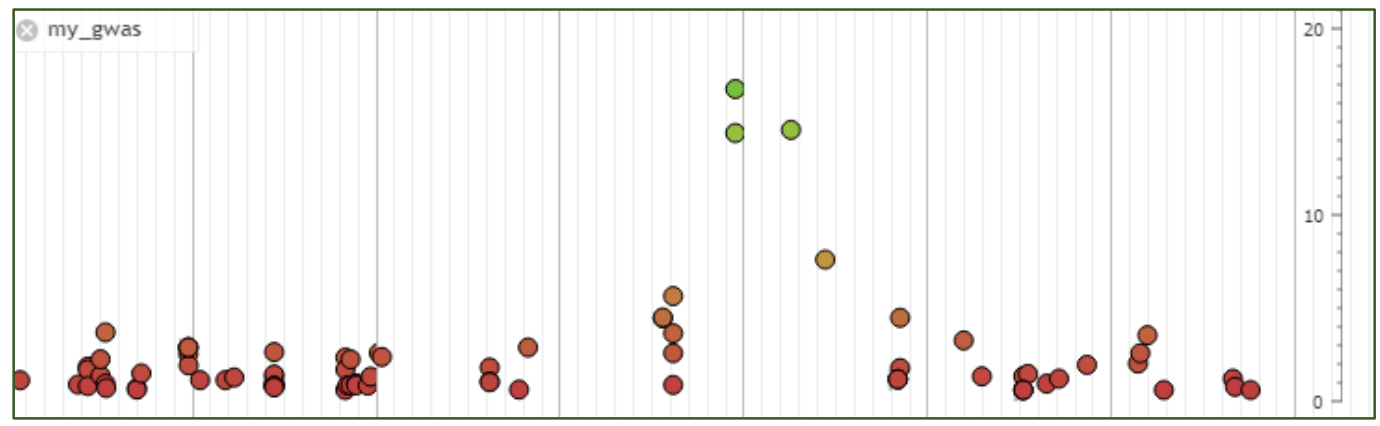

<Figure 26. JBrowse GWAS track >

JBrowse GWAS track. $X$ axis shows Genome coordinate and $t$ axis shows - $\log (p$-value). Each dot represents SNP. Double click the dot to see the ID and coordinate.

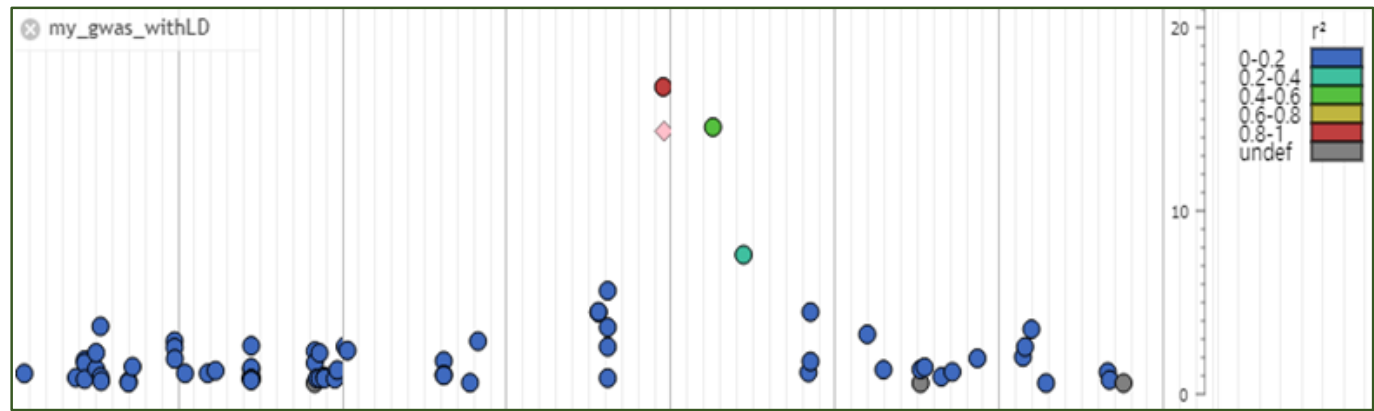

$<$ Figure 27. JBrowse GWAS with LD track >

Uploading GWAS data with LD data will produce default GWAS track (Figure 27). Dot represents SNP. Double clicking the dot will show $\mathrm{R}^{\wedge} 2$ value between other dots (Figure 27). Double clicked dot will be shown as pink diamond.

\section{GWAS}

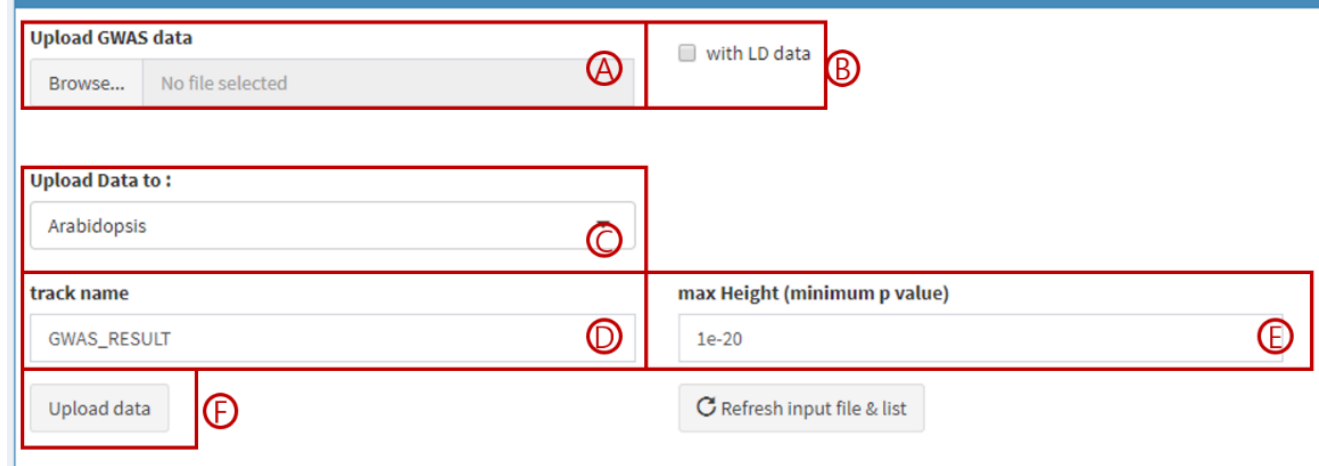

<Figure 28. RNAseq\&GWAS - GWAS > 
(A): Upload IVAG GWAS analysis file.

(B): LD analysis file can be uploaded as well.

(C): Select genome browser to be uploaded.

(D): Set track name.

(E): JBrowse GWAS track's y axis represents - $\log (p$-value). Specify the maximum value for $y$ axis.

(F): Upload GWAS track.

LD

\begin{tabular}{|l|l|}
\hline \multicolumn{2}{|l|}{ Upload LD data } \\
\hline Browse... & No file selected \\
\hline
\end{tabular}

\begin{tabular}{|l|lll|}
\hline \multicolumn{2}{|l|}{ Upload Data to : } & & \multicolumn{2}{l|}{ GWAS track } \\
\hline Arabidopsis & & GFF \\
\hline Upload data & C & & C Refresh input file \& list \\
\hline
\end{tabular}

<Figure 29. RNAseq\&GWAS - LD >

(A): Upload IVAG LD analysis result file.

(B): Select GWAS track to be uploaded.

(C): Click Upload data to add LD information to the specified GWAS track. 